WSRC-TR- $-91-624$

DE92 013365

\title{
EFFECTS OF K-REACTOR PRE-OPERATIONAL COLD FLOW TESTING ON TOTAL SUSPENDED SOLIDS IN PEN BRANCH (U)
}

by

E. W. Wilde

Westinghouse Savannah River Company

Savannah River Laboratory

Aiken, South Carolina 29808

This is a technical report

\section{DISCLAIMER}

This report was prepared as an account of work sponsored by an agency of the United States This report was prepared as an account of work sponsored any agency thereof, nor any of their employees, makes any warranty, express or implied, or assumes any legal liability or responsibility for the accuracy, completeness, or usefulness of any information, apparatus, product, or process disclosed, or represents that its use would not infringe privately owned rights. Referprocess disclosed, or representic commercial product, process, or service by trade name, trademark, manufacturer, or otherwise does not necessarily constitute or imply its endorsement, recommendation, or favoring by the United States Government or any agency thereof. The views and opinions of authors expressed herein do not necessarily state or reflect those of the United States Government or any agency thereof.

This report was prepared in connection with work done under Contract No. DE-AC(09-89SR18035 with the U.S. Department of Energy. By acceptance of this paper, the publisher and/or recipient acknowledges the U.S. Government's right to retain a nonexclusive, royalty-free license in and to any copyright covering this paper, along with the right to reproduce and to authorize others to reproduce all or part of the copyrighted paper. 


\section{DISCLAIMER}

This report was prepared as an account of work sponsored by an agency of the United States Government. Neither the United States Government nor any agency thereof, nor any of their employees, makes any warranty, express or implied, or assumes any legal liability or responsibility for the accuracy, completeness, or usefulness of any information, apparatus, product, or process disclosed, or represents that its use would not infringe privately owned rights. Reference herein to any specific cominercial product, process, or service by the trade name, trademark, manufacturer, or othenvise, does not necessarily constitute or imply its endorsement, recommendation, or favoring by the United States Government or any agency thereof. The views and opinions of authors expressed herein do not necessarily state or reflect those of the United States Government or any agency thereof. 
DBmane - Shehan

D.B.MOORE-SHEDROW, SECTION MANAGER

AUTHORIZED DERIVATIVE CLASSIFIER

EFFECTS OF K-REACTOR PRE-OPERATIONAL COLD FLOW TESTING ON TOTAL SUSPENDED SOLIDS IN PEN BRANCH (U)

BY

EDWARD W. WILDE

WESTINGHOUSE SAVANNAH RIVER COMPANY

SAVANNAH RIVER SITE

AIKEN, SC 29808

PUBLICATION DATE: DECEMBER, 1991

\footnotetext{
APPROVED BY D Brnore Shedres

D.B. MOORE-SHEDROW, MANAGER

ENVIRONMENTAL SCIENCES SECTION

SAVANNAH RIVER LABORATORY
} 


\section{ABSTRACT}

Total suspended solids (TSS) levels were monitored by SRL Environmental Sciences personnel at two locations in the Pen Branch Creek system in conjunction with $K$ Reactor cold flow (pump) testing required as part of the reactor restart effort. The TSS data were compared with flow and rainfall data collected simultaneously in an effort to obtain insight on the suspension and movement of particulate material in the Pen Branch system in response to natural and operational causes. Pump testing clearly caused higher TSS levels at the two sampling locations. The artificially elevated TSS levels were more pronounced at a sampling location near the reactor than at a sampling location farther downstream. Although the environmental data proviled by this study were obtained and used exclusively for process control and research purposes, rather than for formal regulatory compliance (i.e. NPDES monitoring), the TSS levels determined by the comprehensive testing were compared with NPDES limits required at various SRS outfalls. TSS values in Pen Branch were seldom in excess of these limits. Because of the relatively few times that TSS values at the two sampling locations exceeded "typical" NPDES limits, and the fact that occasional relatively high TSS values could clearly be solely attributed to rainfall, it was concluded that no major adverse environmental impacts were caused to the Pen Branch system as a result of the $k$ Reactor pre-operational pump testing. 


\section{CONTENTS}

INTRODUCTION

.1

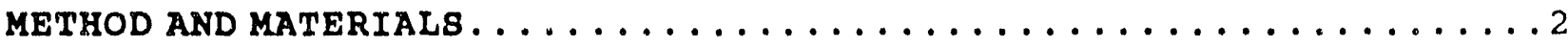

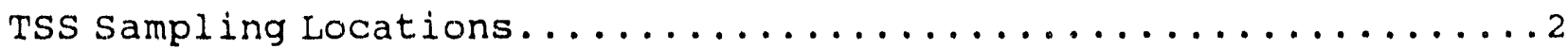

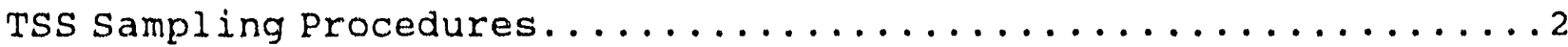

TSS Sample Analysis Procedures....................

Procurement of Ancillary Data....................

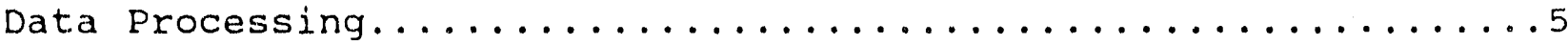

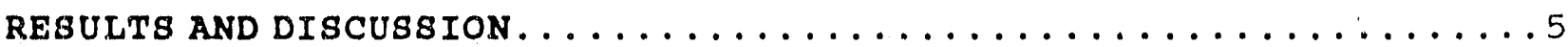

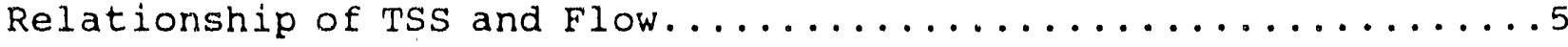

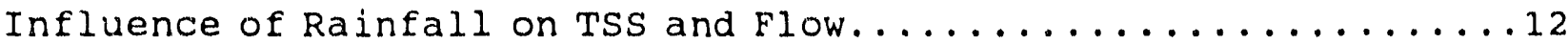

TSS Values in Relation to Compliance Limits

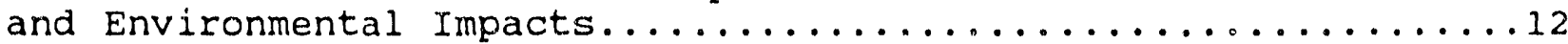

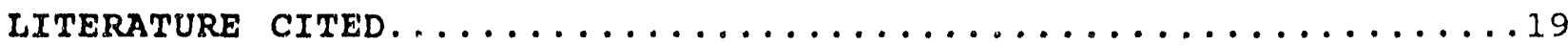

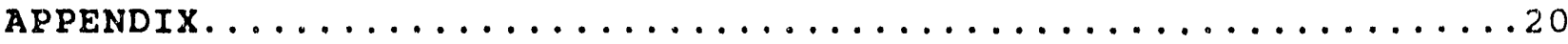




\section{LIST OF FIGURES}

1. TSS Sampling Locations in Relation to $k$ Reactor and the

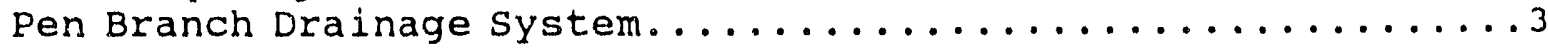

2. Daily Average Flow at Indian Grave Branch above Road $B$ (IGB), within the $\mathrm{K}$-Reactor Cooling water Discharge Canal (K-011, and Downstream Pen Branch at Road A-13.2 (A13.2), ...

3. Scatter Plot Showing Correlations of TSS and Flow Measurements in Pen Branch at Road A-13.2, February through

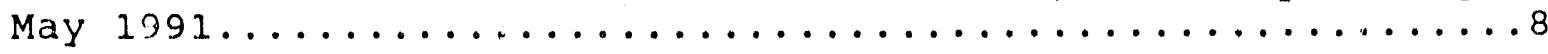

4. Daily Averages of TSS Values Measured in Indian Grave Branch at Road $B$ and in Pen Branch at Road A-13.2,

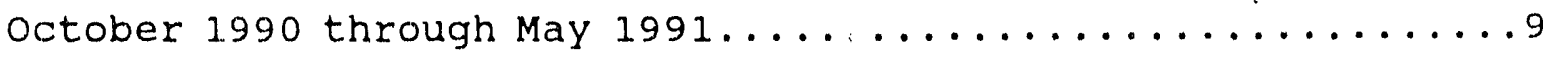

5. Daily Average TSS Values and Flow Measured Simultaneously in Indian Grave Branch at Road B, October 1990 through

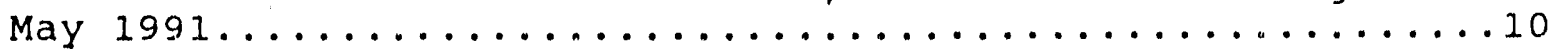

6. Daily Average TSS Values vs. Flow Measured Simultaneously in Pen Branch at Road A-13.2, October 1990 through

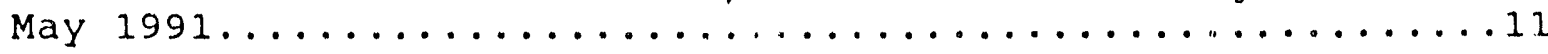

7. Total Daily Rainfall in Relation to Daily Average TSS Values In Indian Grave Branch at Road B ..................

8. Total Daily Rainfall in Relation to Daily Average TSS Values in Pen Branch at Road $A-13.2 \ldots \ldots \ldots \ldots \ldots \ldots \ldots$

9. Total Daily Rainfall in Relation to Daily Average Flow Measured Concurrently with TSS Sampling in Indian Grave

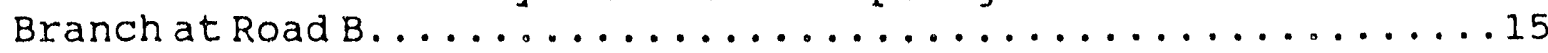

10 Total Daily Rainfall in Relation to Daily Average Flow Measured Concurrently with TSS Sampling in Pen Branch at

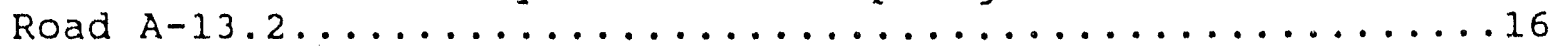




\section{LIST OF TABLES}

1. Monthly Average and Daily Maximum (in Parentheses) Total Suspended Solids (mg/l) and Flow (cfs) at Two Locations of the Pen Branch System before and during $K$ Reactor Pump Testing. Total Inches of Rainfall Recorded in K Area Are Also Reported for Each Month........ 17

A1. Total suspended Solids (TSS) and Flow in Pen Branch at Road A-13.2, October 1990 through May 1991.............21

A2. Total suspended Solids (TSS) and Flow in Indian Grave Branch at Road B, October 1990 through May 1991..........42

A3. Rainfall in $K$ Area in Relation to Total suspended Solids and Corresponding Flows at Two Locations in the Pen Branch system................................64

A4. USGS Flow Measurements (Provisional Data). Daily Averages at Three Gaging Stations; Indian Grave Branch above Road $B$ (IGB), K-Reactor Cooling water Discharge Canal ( $K-011)$, and

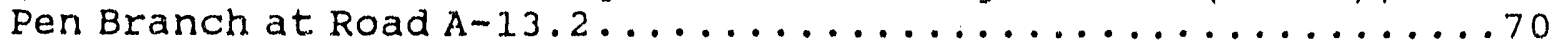




\section{INTRODUCTION}

Preparation for restarting $K$ Reactor required testing of numerous cooling water flow and pumping systems. The testing required that ambient temperature water be released from the reactor's secondary cooling water outfall and discharge canal to Indian Grave Branch, a tributary of Pen Branch. Intermittent testing of emergency cooling system pumps and pumps providing water to the heat exchangers, combined with periods of gravity flow (which can be higher than running one or two pumps) resulted in the discharge of water at a variety of flow rates for a variety of frequencies during the study period, which ranged from october 1990 through May 1991. The majority of the pump testing activity occurred between February and April of 1991. Thus, the environmental sampling conducted as part of this study overlapped the majority of pump tests and allowed an assessment of environmental monitoring data attributable to natural factors such as rainfall and runoff to be compared with values primarily attributable to the pump tests.

Total suspended solids (TSS) was one of the parameters selected to be monitored by the SRL Environmental Sciences section (ESS) during the principal period in which pump testing was conducted and is the focus of this report. A report describing the impact of the pump testing on the fish community of Pen Branch has been prepared by Paller (1991). One of the reasons that TSS was selected for monitoring was because such data can be obtained at frequent intervals and reported relatively quickly. This allowed monitoring data obtained by ESS to be used in the design of the pump testing while it was in progress to aid in the protection of the biotic communities in Pen Branch.

The term total suspended solids (TSS) is synonymous with the terms "non-filterable residue" and "dry weight" and essentially is the combination of organic and inorganic particulate material that is retained on a glass fiber filter dried to a constant weight of 103$105^{\circ} \mathrm{C}$. This parameter gives an indication of the flow and sediment transport related impacts to a stream ecosystem, particularly the fish and aquatic invertebrates. Water quality criteria proposed by the U.S. EPA require that "settleable and suspended solids should not reduce the depth of the compensation point for photosyntratic activity by more than 10 percent from the seasonably established norm for aquatic life" (U.S. EPA, 1986). It was not practical to measure compensation point depths in the Pen branch system and no specific numerical TSS values for protection of aquatic life have been established by the EPA. However, NPDES permits typically contain TSS limits for point source discharges. At SRS, these limits typically include daily maximum values of $60 \mathrm{mg} / \mathrm{l}$ and/or monthly mean values of $30 \mathrm{mg} / \mathrm{l}$ at many outfalls (state of south Carolina, 1984). 
TSS sampling involved the collection of water by automatic portable samplers programmed to collect discrete samples at pre-determined intervals, typically every four hours when pump testing was not known to be in progress and more frequently when pump testing was known to be in progress. This sampling strategy and equipment allowed collections to be made in a consistent manner during all parts of the day and night as well as during inclement weather. However, the use of unattended samplers resulted in some problems such as sampling gear being dislodged by logs traveling downstream, etc. These events are undoubtedly responsible for some anomalies in the resulting data.

To aid in the interpretation of results from the TsS sampling, hourly flow data (provisional) for gaging stations nearest the TSS sampling locations were obtained from the U.S. Geological survey (USGS) and daily rainfall data recorded at $k$ Area were obtained from the SRL Environmental Technology Section's Environmental Transport Group. This supplemental information was used in determining periods when pump tests affected flow in the stream system and differentiating between TSS quantities primarily attributable to the pump testing and TSS quantities partially or fully attributable to natural factors such as rainfall and runoff.

\section{METHOD AND MATERIALS}

\section{Tss Sampling Locations}

Two locations in the Pen Branch watershed were selected for monitoring of TSS (Figure 1). Both locations are downstream from the $K$ Reactor cooling water discharge point. The station on Indian Grave Branch at Road $B$ is below the confluence of The K-Reactor cooling water discharge canal with Indian Grave Branch, approximately $700 \mathrm{~m}$ below the discharge pipe. The station on Pen Branch at Road $A-13.2$ is below the confluence of Indian Grave Branch and upper Pen Branch, approximately $6.2 \mathrm{~km}$ below Road B.

\section{TSS Sampling Procedures}

Portable, automatic sequential samplers (Isco and/or Manning) housing 24 bottles each were placed on a catwalk adjacent to the stream locations described above. Approximately $8 \mathrm{~m}$ of tubing attached to each sampler was positioned at approximately midstream and middepth using floats and weights. The samplers were programmed to collect water samples of approximately $400 \mathrm{ml}$ each at intervals which ranged in frequency from daily to hourly. Generally the samplers were programmed to collect four samples per day at sixhour intervals. When all, or in some cases most, bottles within a sampler contained samples, the bottles were exchanged with empty ones and the sample containing bottles were returned to the lab for processing. 


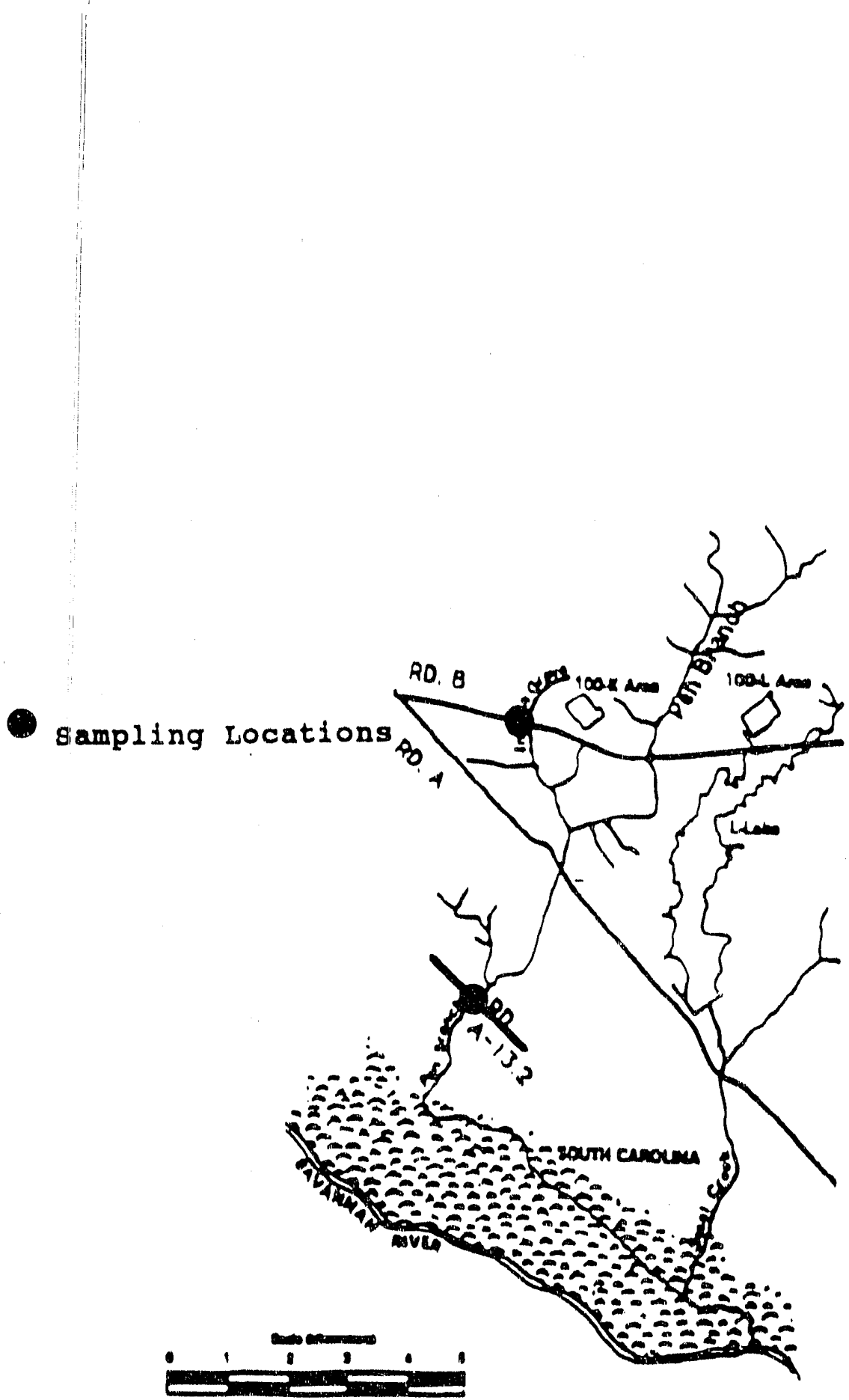

Figure 1. TS8 sampling Locations in Relation to R Reactor and the Pen Branch Drainage system. 


\section{T8s sample Analysis procedures}

TSS sample processing and analysis procedures were performed by trained personnel in accordance with SRL Environmental sciences Section Procedure 2-48 that parallels EPA Method 160.2 for nonfilterable residue (U.S. EPA, 1979). The contents of all sample bottle were filtered within one hour of the time they were returned to the laboratory. Whatman $47 \mathrm{~mm}$ diameter GF/C glass fiber filter disks were used. Prior to use, the filters were labeled and prewashed three times with $20 \mathrm{ml}$ of deionized water. The clean filters were dried at $103-105^{\circ} \mathrm{C}$ and placed in a desiccator to cool to room temperature. After a constant weight of a washed and dried filter was obtained and recorded, the filter was subjected to use with a sample of stream water. A $100 \mathrm{ml}$ aliquot from a vigorously shaken sample bottle was filtered using a Millipore filtering apparatus which included a suction flask, vacuum pump, and coarse fritted disk filter support. After filtering, the filter was dried at 103$105^{\circ} \mathrm{C}$ for one hour and cooled in a desiccator until a constant weight was obtained. All samples were analyzed in duplicate. TSS values were calculated by the following formula:

$$
\begin{aligned}
& \text { TSS in } \mathrm{mg} / \mathrm{I}=\frac{(\mathrm{A}-\mathrm{B}) \times 1000}{\mathrm{C}} \\
& \text { Where: } \\
& \mathrm{A}=\text { weight of filter + residue in mg (mean of two } \\
& \text { weighings) } \\
& B=\text { weight of filter (mean of two weighings) } \\
& C=\text { ml of sample filtered }
\end{aligned}
$$

A11 TSS data obtained from the study were recorded in logbook WSRCNB-90-329 and are recorded on electronic media (computer files) being maintained by E.W. Wilde.

\section{Procurement of Anciliary Data}

Provisional flow data were obtained from the USGS which maintains flow monitoring stations at the Road A-13.2 location, SRS NPDES outfall K-011 located in the K Reactor discharge canal, and Indian Grave Branch located above Road B. Unfortunately, the latter station was not operational for much of the time during the present study. To estimate the total flow in Indian Grave Branch at Roac. B, we added the flow recorded at $\mathrm{K}-01 \mathrm{l}$ with the flow recorded at the upstream Indian Grave Branch location. When no data were available for the upstream Indian Grave Branch gaging station, a correction factor of $0.4 \mathrm{cfs}$, an approximate long term average for this location (Larry Moray, USGS, personal communication) was added to the K-011 flow value to obtain an estimate of flow at Road B. Daily rainfall records for $K$ Area were obtained from the SRL Environmental Technology section. 


\section{Data Processing}

All TSS and flow data along with pertinent other ancillary data were entered into a Lotus $1-2-3$ data set. Data processing and graph preparation were conducted using Lotus 1-2-3 and Harvard Graphics computer software with an IBM PC.

\section{RESULTS AND DISCUSSION}

Tabular summaries of the hourly and average values (daily and monthly) from TSS and flow measurements at Road A-13.2 and Road $B$ are included in Appendix Tables $A 1$ and $A 2$. Daily average TSS and flow values along with daily precipitation measured in $K$ Area are listed in Appendix Table A3. Appendix Table A4 lists daily average flow data (provisional) for the three gaging stations in the pen Branch drainage system maintained by the USGS. Slightly different daily averages flow values between Appendix Table A4 compared to the other Appendix Tables are a result of averaging 24 hourly flow measurements for a daily average in Appendix table A4, while only flow measurements taken concurrently with TSS measurements were used to obtain the daily average flow measurements in Appendix Tables Al-A3.

Figure 2 shows the daily average flow data presented in Appendix Table A4. The relatively minor contribution of flow from upstream Indian Grave Branch (station IGB) is obvious, even though this gaging station was not operational during much of the study period. It is also clear that the flow at outfall K-011, principally consisting of $k$ Reactor cooling water discharges, is very closely linked to the flow in Pen Branch at Road A-13.2. However, exceptions occur during heavy rainfall. Four peaks of flow at Road A-13.2 that were not accompanied by corresponding peaks of flow at Outfall K-011 occurred in October (mid and late), late January, and the beginning of March. Heavy rainfall occurred during each of these periods. (Appendix Table A3).

\section{Relationship of Tss and Flow}

At the Road A-13.2 location, 603 TSS measurements were made. The average TSS concentration was $17 \mathrm{mg} / 1$ with a range of 0.5 to 145 $\mathrm{mg} / \mathrm{l}$. There were 626 measurements of TSS at the Road B Location. The average value was 17.5 with a range of 0.5 to $268.5 \mathrm{mg} / \mathrm{l}$.

Specific flow values corresponding to the TSS values were obtained from the hourly USGS monitoring data as previously described. These flow values averaged $159 \mathrm{cfs}$ and $193 \mathrm{Cfs}$ at Road $B$ and Road A-13.2, respectively. At Road $B$, flow ranged from 29 cfs to 484 cfs while at Road $A-13.2$, flow ranged from 34 cfs to 833 cfs during periods that samples for TSS were collected. 


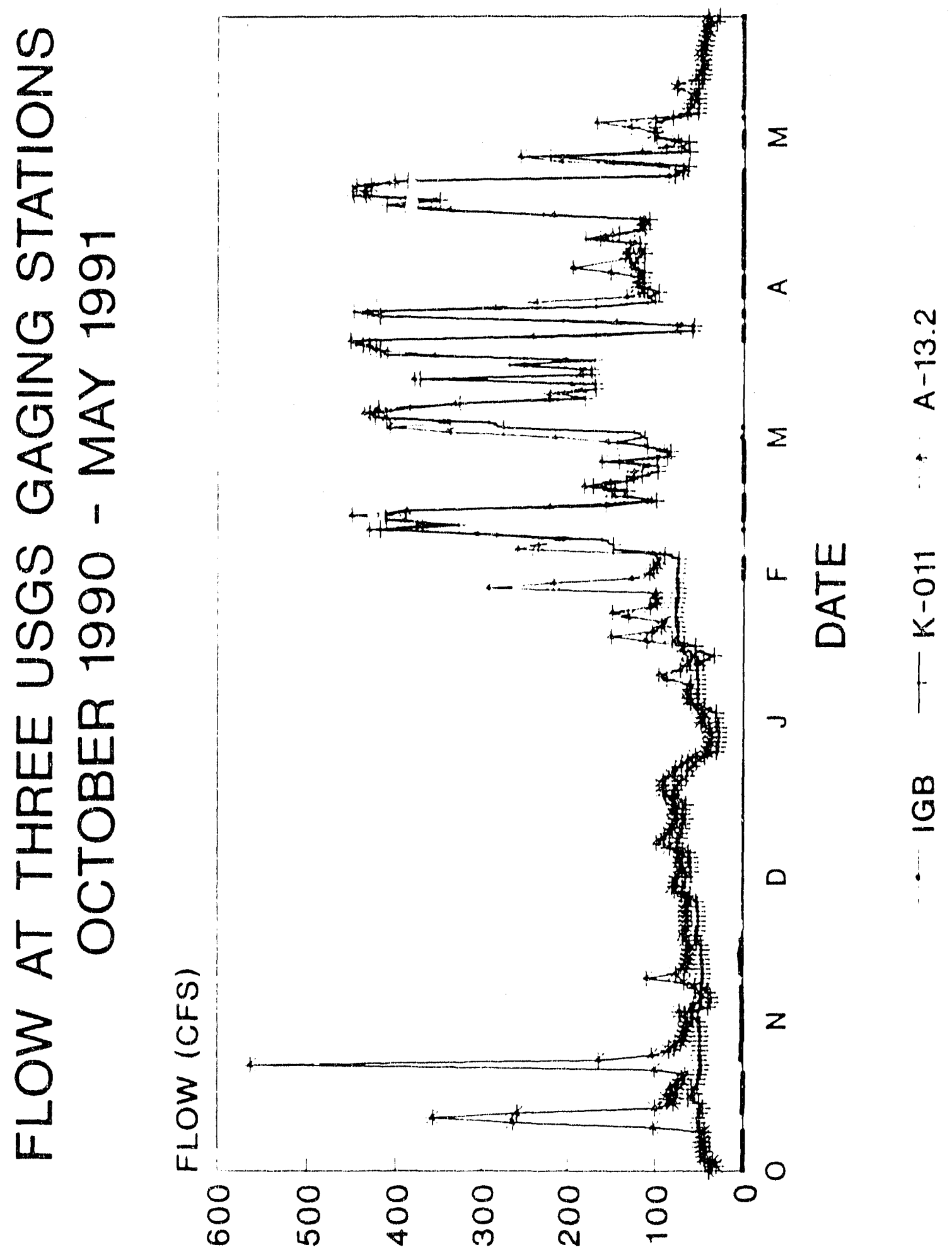

Figure 2. Daily Avarage Flow at Three Locations of the Pen Branch Drainage 8ystem: Indian Grave Branch above Road B (IGB), within the $R$-Reactor Cooling water Dischargo Canal ( $R$ 011), and Downstream Pen Branch at Road $A-13.2(A-13.2)$. 
The relationships between flow and TSS were not as distinctive as was expected. Correlation coefficients between flow and TSS values were statistically significant $(r=0.45$ and 0.22 , at the Road $B$ and Road $A-13.2$ locations, respectively) but not strong. Figure 3 shows a scatter plot matrix illustration of TSS data plotted against flow at the Road $A-13.2$ location for data collected from February through May 1991, the period encompassing most of the pump testing. The data contain numerous outliers for which no explanation is obvious. Because of the extreme variability in TSS in relation to flow at specific times, The data were primarily analyzed in terms of daily average values.

Figure 4 shows daily average TSS values during the study period. There were four distinct peaks of TSS values. The first peak occurred in october and appeared to be caused by heavy rainfall. The second peak occurred in late November. This peak occurred at only the Road A-13.2 location and is believed to be the result of a sampling problem when a transient log dislodged the intake hose of the sampling device. The third and fourth peaks of TSS values occurred in mid-February and mid-March and appeared to be primarily caused by the pump testing.

Figures 5 and 6 show TSS values in relation to correrponding flows at the two sampling locations. With the exception of the previously discussed anomalous TSS peak in November at Road A-13.2, flows and TSS values tend to follow similar patterns. The first surges in flow attributable to the pump tests in February and March resulted in much higher corresponding TSS values than did subsequent flow surges of similar magnitude. This finding resulted in a decision which allowed the pump tests to be conducted more quickly by rapidly increasing and decreasing flow as routine pump test protocols dictated, rather than being restricted to stepwise increases in flow as a precaution against causing TSS related adverse impacts to the stream biota during the tests.

Consistent relatively high TSS values at Road A-13.2 during late May did not correspond to high flows, heavy rainfall or high TSS or flow values at Road $B$ during this period. Two possible explanations for these results are that work was being done on the railroad bridge a short distance above the Road A-13.2 sampling location during this period and there were ongoing activities associated with the K-Reactor cooling tower. Either or both of these activities may have resulted in an increased load of particulate material during the late May sample collections. Cooling tower construction may have also been a factor in some of the other relatively high TSS values that were reported at the Road A-13.2 location with no corresponding increased flow. 


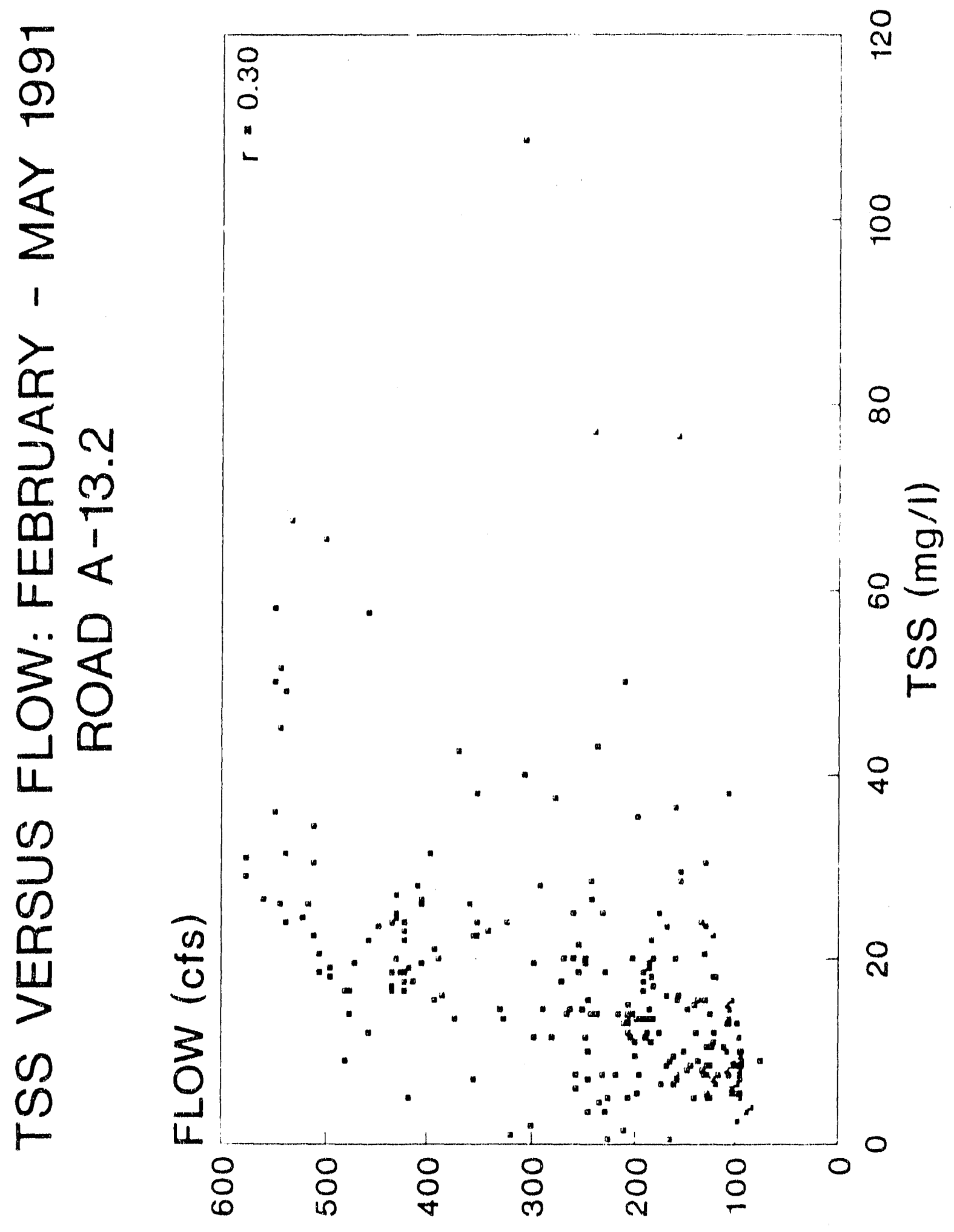

Figure 3. Scatter Plot showing correlations of Ts8 and Flow Measurements in Pen Branch at Road A-13.2, February through May 1991. 


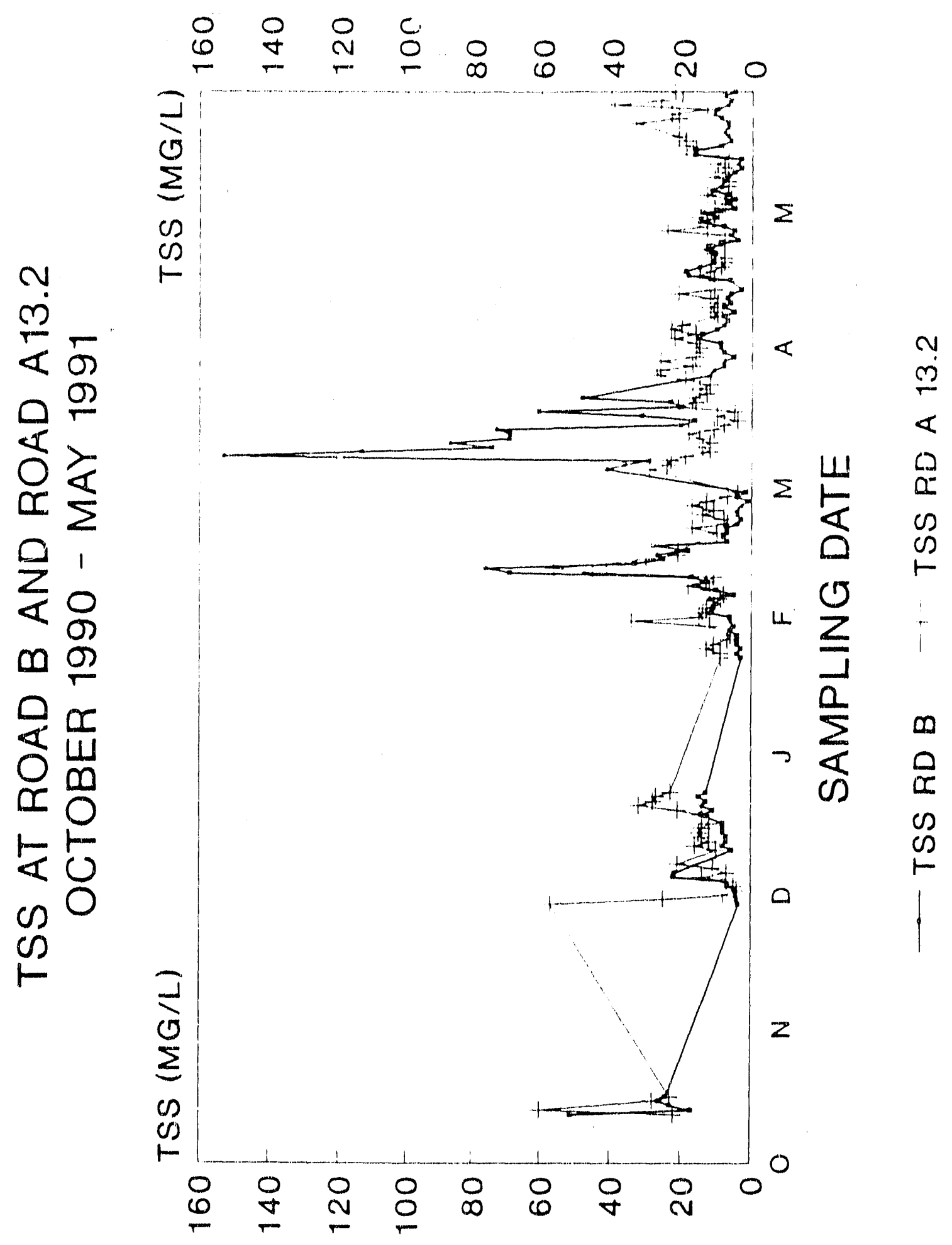

Figure 4. Daily Averages of TSS values Measured in Indian Grave Branch at Road $B$ and in Pen Branch at Road A-13.2, october 1990 through May 1991. 


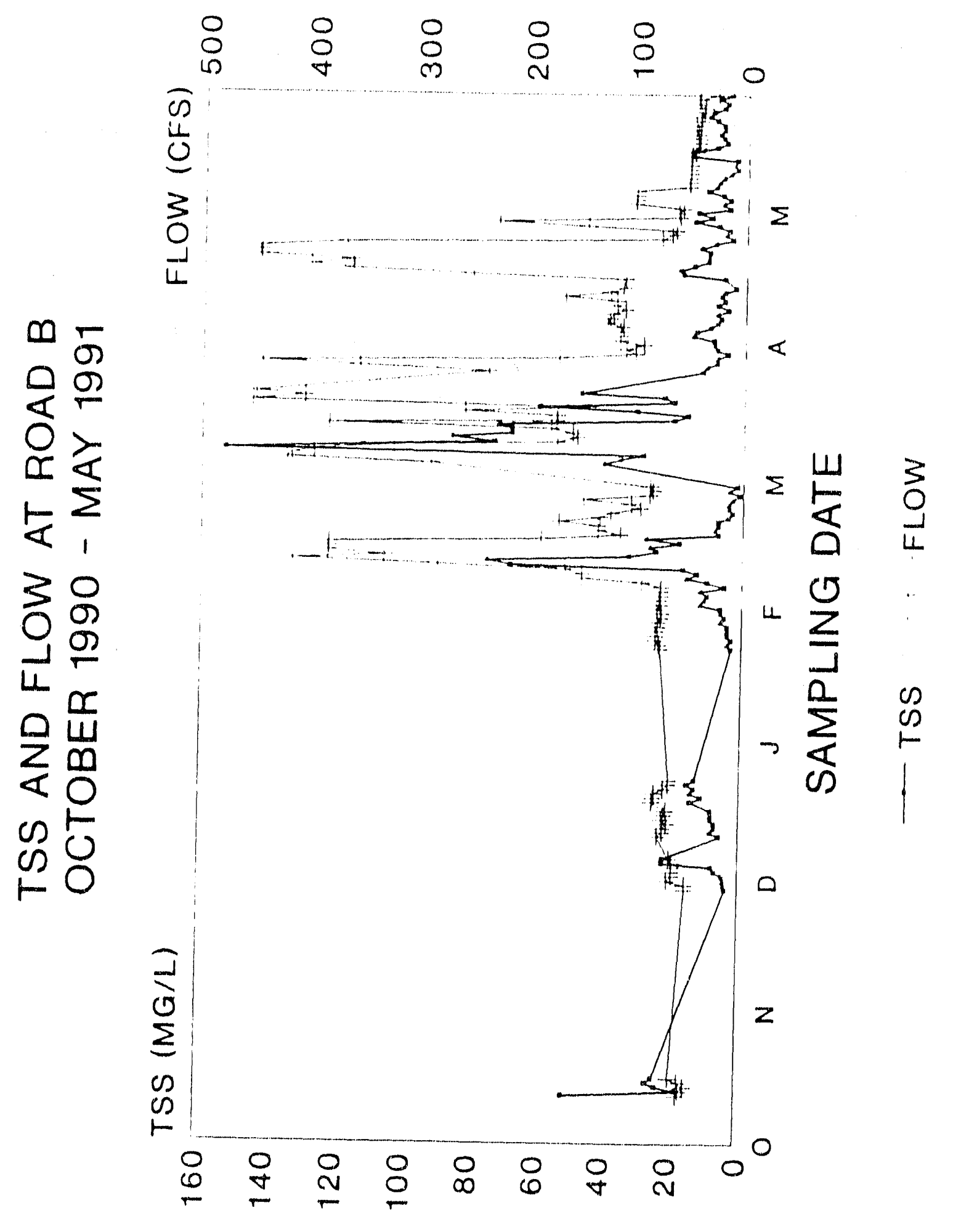

Figure 5. Daily Average Ts8 Values vs. Flow Measuxed simultaneously in Indian Grave Branch at Road B, October 1990 through May 1991. 


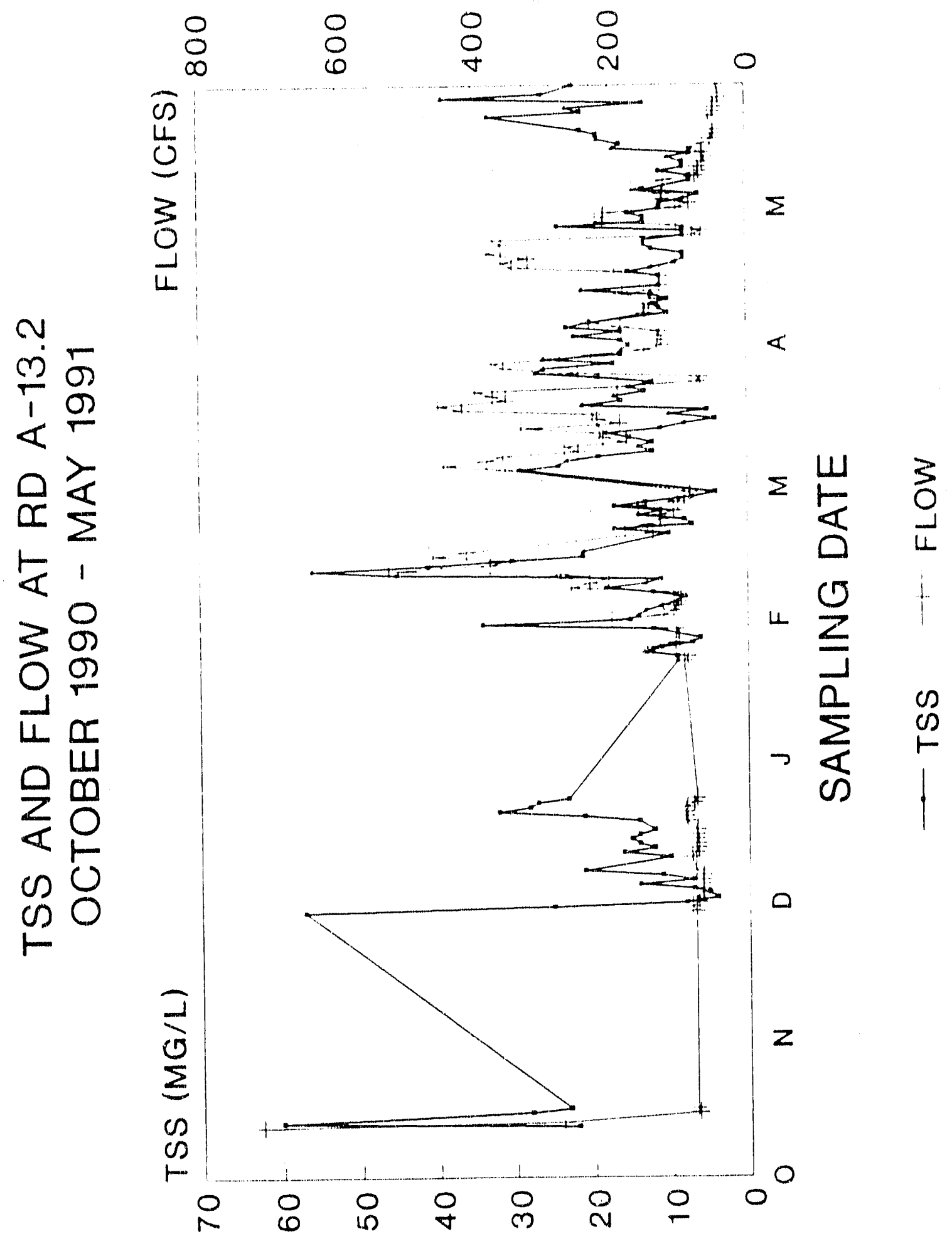

Figure 6. Daily Average Tss Values vg. Flow Measured simultaneougly in Pen Branch at Road A-13.2, October 1990 through May 1991. 


\section{Influence of Rainfall on TSS and Flow}

Figures 7 and 8 show total daily rainfall in relation to daily average TSS values at the two sampling locations. Figures 9 and 10 show a similar comparison of rainfall in relation to flow. It is clear that the high TSS values in october were caused by heavy rainfall ( 9.74 inches in three days; Appendix Table $A-3$ ) that preceded the peaks. High flow and TSS levels at the Road A-13.2 location in late January also appeared to be caused by heavy rainfall (Appendix Table $A-3$ ). Conversely, the TSS peaks in February at Road $A-13.2$ and at Road $B$ in February and March did not appear to be substantially influenced by rainfall since higher rainfall during other periods resulted in much lower Tss values. Rainfall was more strongly correlated with flow at Road A-13.2 than at Road $B$. However, because of the flow increases caused by the pump tests, neither station showed a distinct relationship between rainfall and flow during much of the study period.

TsS values in Relation to Compliance Limits and Environmental Impacts.

This study was not conducted as a regulatory monitoring/compliance activity and the sampling was not conducted at specifically designated NPDES outfall sampling locations. However, for comparative purposes, the data were processed and examined similarly. NPDES limits for TSS are based on daily maximum and monthly average values. The daily maximum is defined as the highest daily average during a month, while the monthly average is defined as the arithmetic mean of all samples collected during a month. The NPDES effluent limitation value that is most relevant to this study is a daily maximum of $60 \mathrm{mg} / \mathrm{l}$, the upper acceptable limit proposed for the future K-Reactor cooling tower blowdown outfall which will be designated as NPDES Outfall k-0.18. This station will be very close to the Road $B$ sampling location of the present study. Although the present draft permit does not stipulate a monthly mean TSS limit for outfall $\mathrm{K}-018$, several other SRS outfalls are required to meet a limit of $30 \mathrm{mg} / 1$. Thus, for comparative purposes, values from the present study were compared to "typical" NPDES limits which are assumed to represent levels that would not result in any significant adverse impact if not exceeded.

The monthly mean and daily maximum TSS values obtained from the present study along with corresponding flow values and the amount of precipitation received during each month of the study are shown in Table 1. The monthly mean TSS values at the two locations reached or exceeded "typical" NPDES limits in March at the Road B location and in october at the Road A-13.2 location. As previously discussed, pump testing was a substantial factor in the relatively high March value and not a factor in the high monthly mean TSS value for october. However, both October and March had considerable 


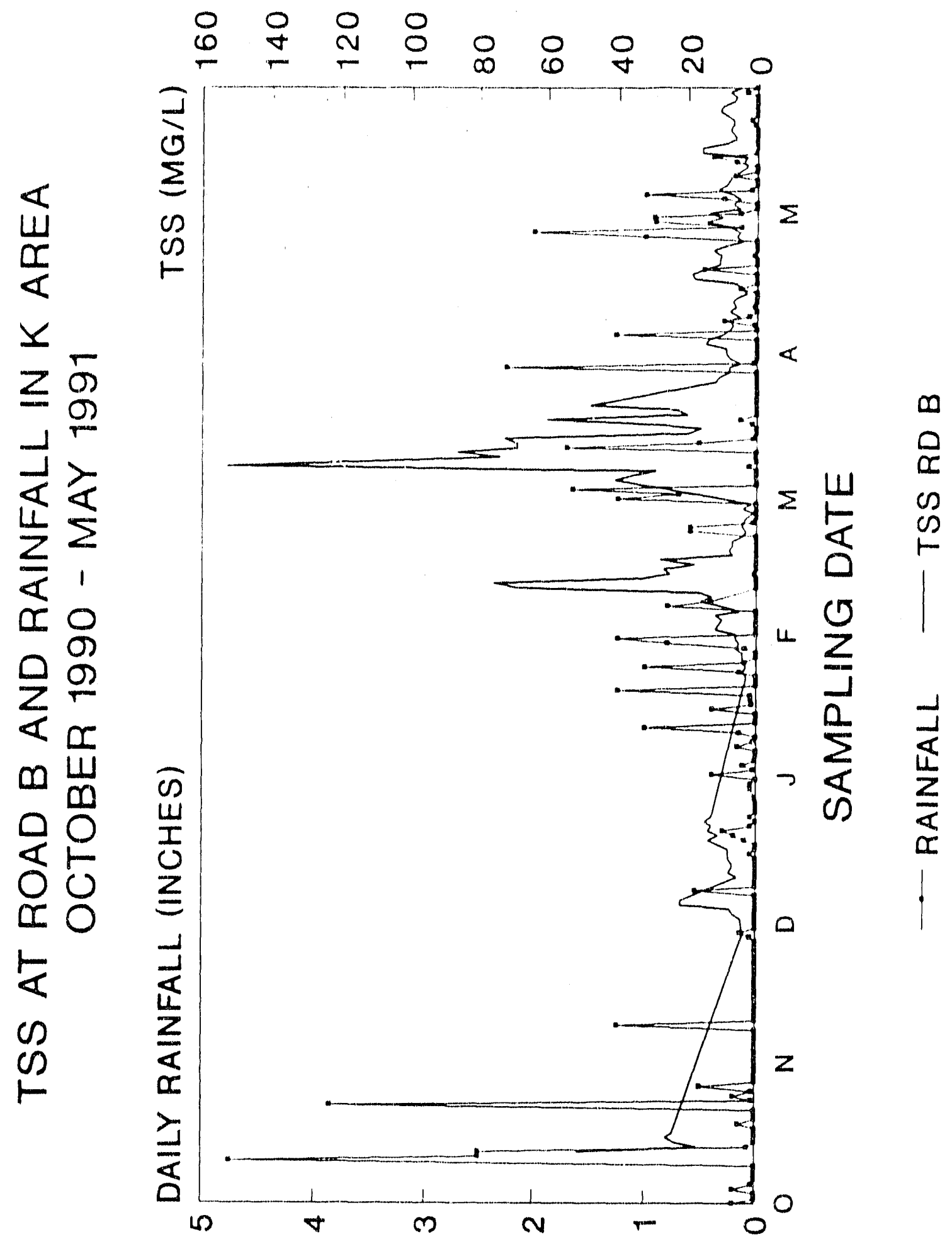

Figure 7. Total Daily Rainfall in Relation to Daily Average T88 values In Indian Grave Branch at Road B. 


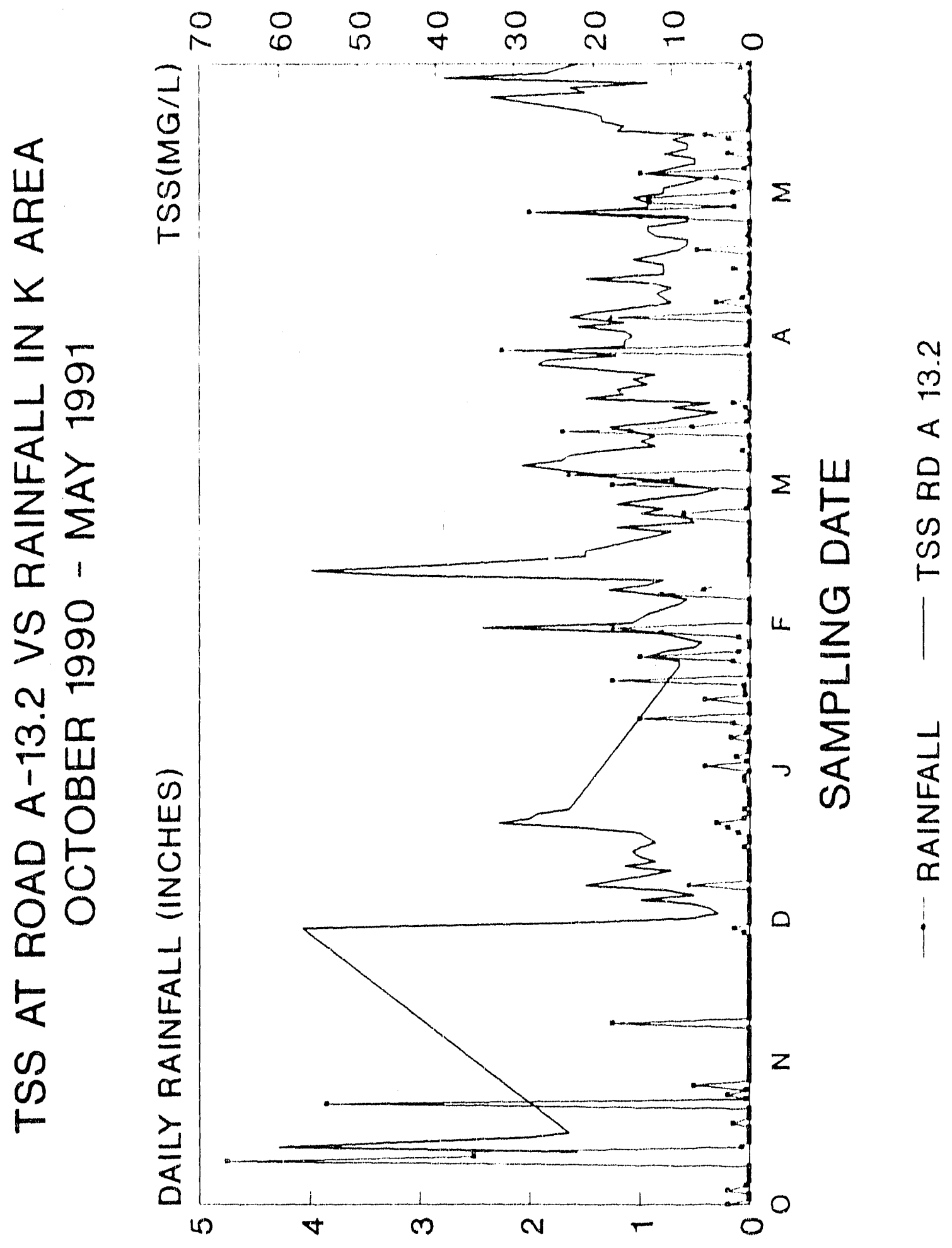

Flgure 8. Total Daily Rainfall in Relation to Daily Average TSB values in Pen Branch at Road A-13.2. 


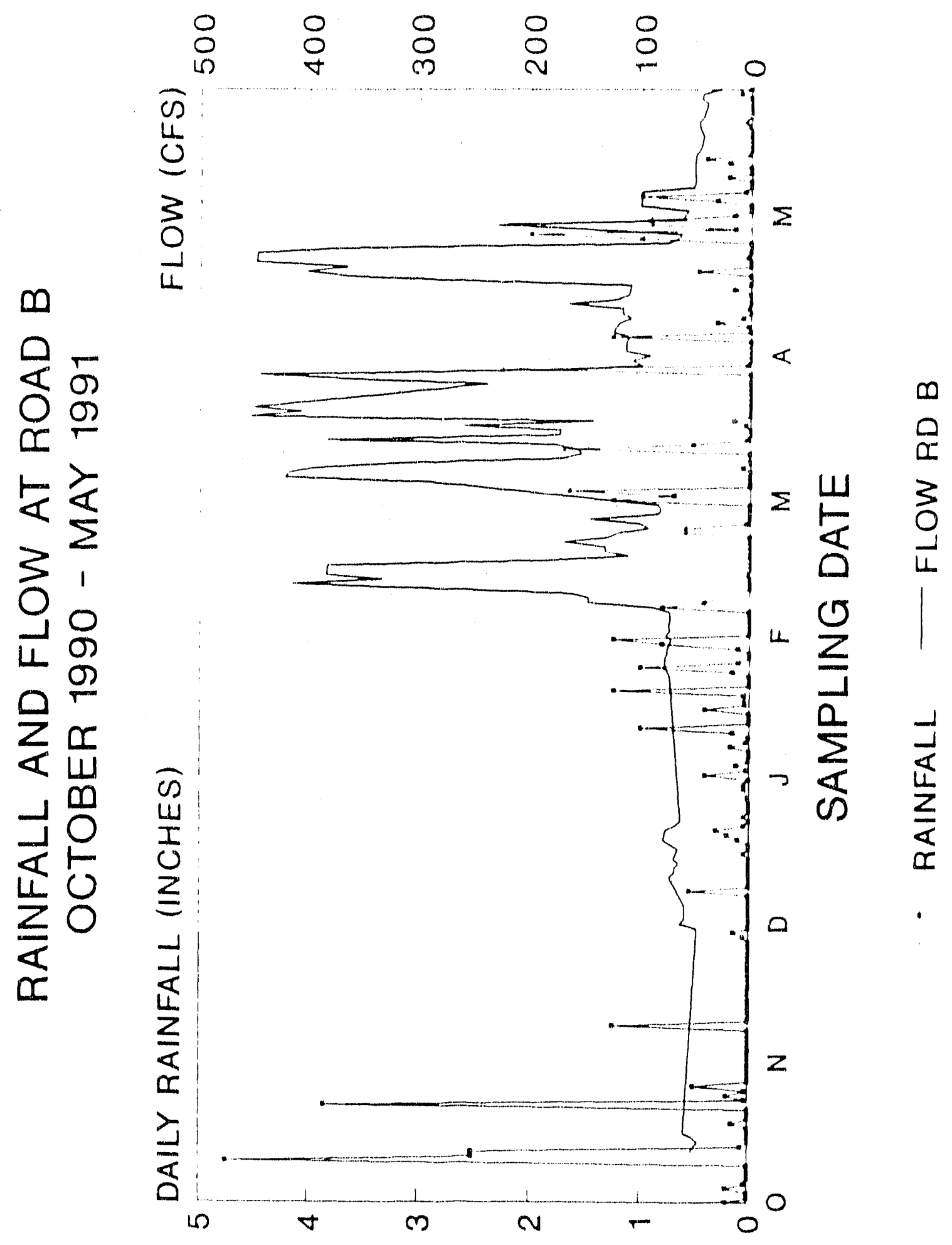

Figure 9. Total Daily Rainfall in Relation to Daily Average Flow Measured Concurrently with Ts8 sampling in Indian Grave Branch at Road B. 


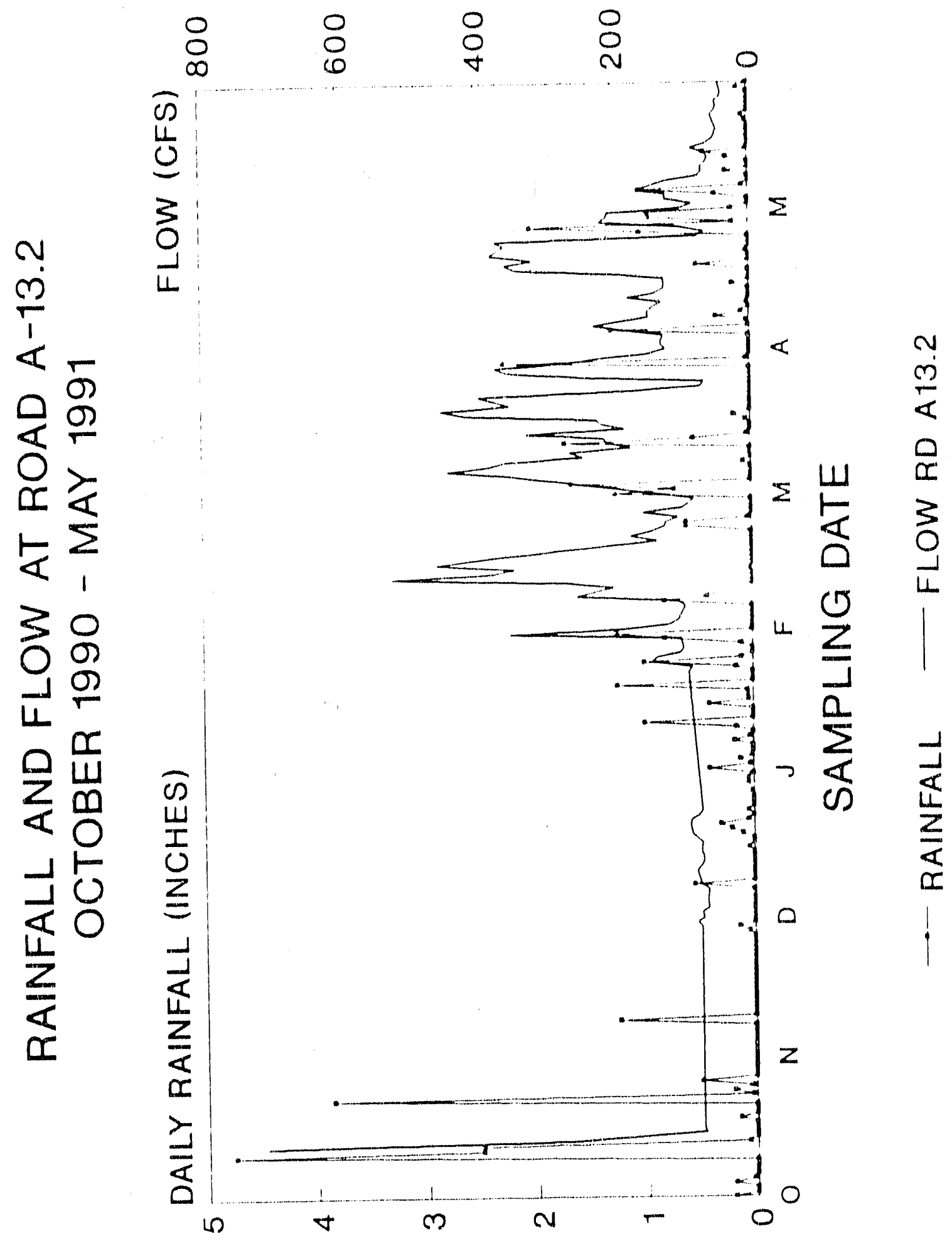

Figure 10. Total Daily Rainfall in Relation to Daily Average Flow Measured Concurrently with TS8 sampling in Pen Branch at Road $A-13.2$. 
Table 1. Monthly Average" and Dally Maximum (in Parenthases) "Total suspended solids (mg/l) and Flow (cfs) at Two Locations of the Pen Branch system before and during $k$ Reactor Pump Testing. Total Inches of Rainfall Recorded in $K$ Area Are Also Reported for Each Month.

TOTAL SUSPENDED SOLIDS DISCHARGE (FLOW) PRECIPITATION

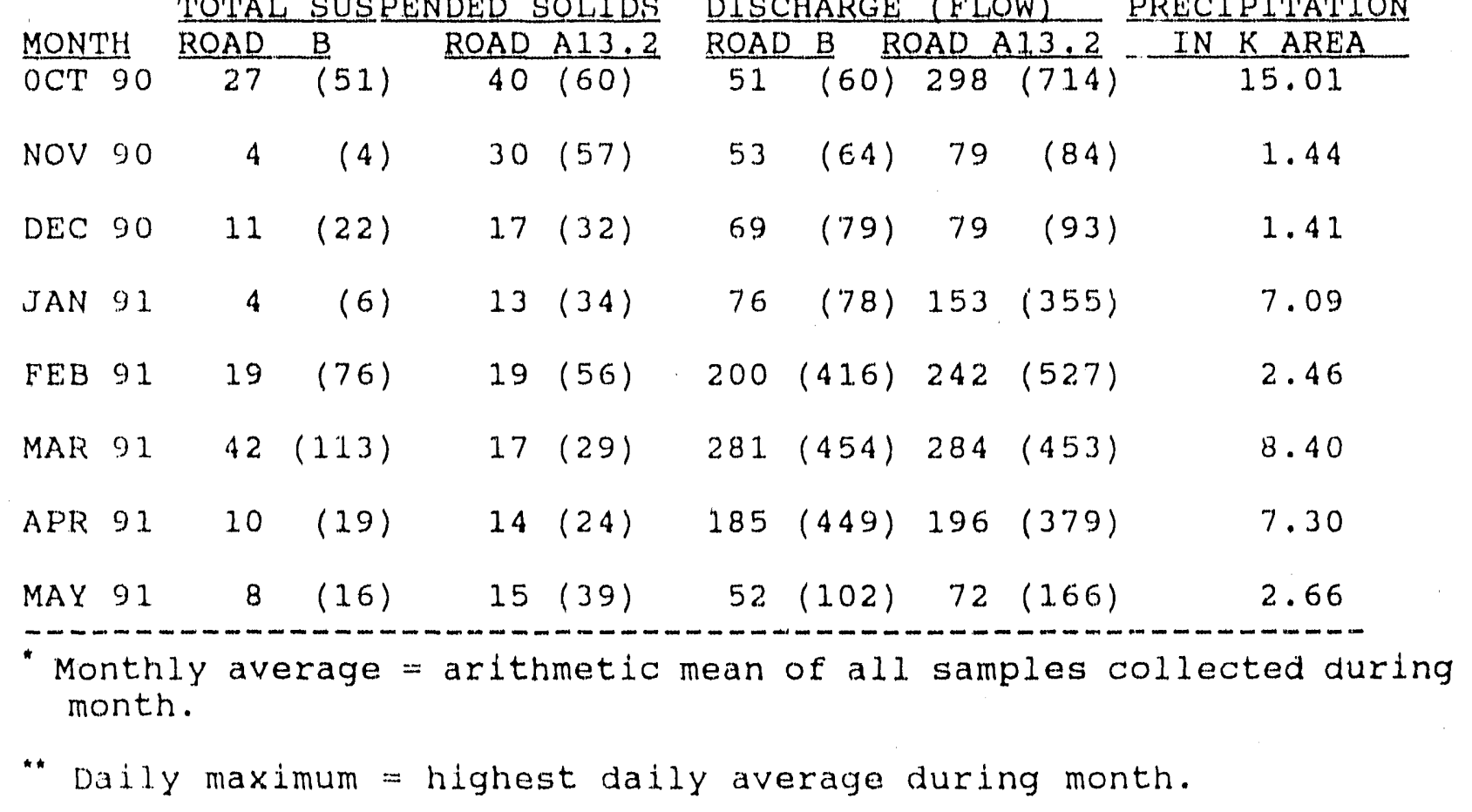


rainfall, 15.01 and 8.40 inches, respectively. Daily maximum 'TSS values equalled or exceeded $60 \mathrm{mg} / 1$ in February and March at the Road B location and in October at the Road A-13.2 location. These values corresponded with the highest flow values recorded during the study. Again, the relatively high TSS value in october appeared to be entirely caused by substantial rainfall, while the relatively high vaiues for February and March were caused by a combination of rainfall and pump testing, with pump testing being the primary factor.

Fl sw at Road $B$ was more indicative of pump test activity than flow at Road $A-13.2$ because a much higher percentage of the flow at Road $B$ comes from K-Reactor cooling water discharges. Flow values at Road $B$ did not correspond to rainfall values nearly as much as did the flow values at Road A-13.2. The flow at Road A-13.2 was highest in october during a rain storm (when no pump testing was being conducted) than at any other time during the study, while the flow at Road $B$ was highest in February, March and April, the period that most of the pump testing was conducted.

In summary, pump testing clearly caused higher TSS levels at the two sampling locations in the Pen Branch system. The artificially elevated TSS levels were more pronounced at the Road B location than at the farther downstream Road $A-13.2$ location. However, the relatively few times that Tss values at the two sampling locations exceeded "typical" NPDES limits and the ocuasional relatively high TSS and flow values that could be clearly attributed to rainfall alone indicated that no major adverse environmental impacts were caused to the stream system as a result of the pump testing. 


\section{LITERATURE CITED}

Paller, M.H., Modica, S. and Hofstetter, E.G. 1991. Impact of artificial increases in flow on fish assemblages in two southeastern streams. Rivers (in prep.)

State of South Carolina, 1983. NPDES Permit No. SC0000175 issued December. 15, 1883 to the U.S. Department of Energy, Savannah River Plant. Effective date January 1, 1984.

U.S. Environmental protection Agency, 1986. Quality Criteria For Water, 1986. EPA 440/5-86-001.

U.S. Environmental. Protection Agency. 1979. Methods For Chemical Analysis of Water and wastes. EPA-600/4-79-020. 
APPENDIX 
Appendix Table A1. Total suspended solids (TSS) and Flow in Pen Branch at Road A-13.2, October 1990 through May 1991.

\section{DATE}

$10 / 12 / 90$

$10 / 12 / 90$

$10 / 12 / 90$

$10 / 12 / 90$

$10 / 13 / 90$

$10 / 13 / 90$

$10 / 13 / 90$

$10 / 13 / 90$

$10 / 13 / 90$

$10 / 13 / 90$

$10 / 13 / 90$

$10 / 15 / 90$

$10 / 15 / 90$

$10 / 15 / 90$

$10 / 15 / 90$

$10 / 16 / 90$

$10 / 16 / 90$

$10 / 16 / 90$

$10 / 90$

$11 / 28 / 90$

$11 / 29 / 90$

$11 / 30 / 90$

$11 / 90$

$12 / 1 / 90$

$12 / 2 / 90$

$12 / 3 / 90$
TIME

1030

1830

2230

MEAN

230

630

1030

1430

1830

2230

MEAN

1300

1700

2100

MEAN

100

500

MEAN

AVE.

1730

1730

1730

AVE

1730

1730

1730
T88

(MG/L)

21

29

16

22

12

12

21

95

145

77

60

41

23

22

28

19

27

23

40

57

25

8

30

4

5

7
69

FLOW

(CFB)

594

833

714

714

637

373

248

166

127

99

275

81

72

72

75

78

78

78

298

78

84

74

79

78

67 
APPENDIX TABLE A1. (CONT.)

\section{DATE}

$12 / 4 / 90$

$12 / 4 / 90$

$12 / 4 / 90$

$12 / 5 / 90$

$12 / 5 / 90$

$12 / 5 / 90$

$12 / 5 / 90$

$12 / 5 / 90$

$12 / 6 / 90$

$12 / 6 / 90$

$12 / 6 / 90$

$12 / 6 / 90$

$12 / 6 / 90$

$12 / 7 / 90$

$12 / 7 / 90$

$12 / 7 / 90$

$12 / 10 / 90$

$12 / 10 / 90$

$12 / 10 / 90$

$12 / 11 / 90$

$12 / 11 / 90$

$12 / 11 / 90$

$12 / 11 / 90$

$12 / 12 / 90$

$12 / 12 / 90$

$12 / 12 / 90$

$12,12 / 90$

$12 / 12 / 90$

$12 / 13 / 90$

$12 / 13 / 90$

$12 / 13 / 90$

$12 / 13 / 90$

$12 / 13 / 90$
TIME

1400

2000

MEAN
200
800
1400
2000

MEAN

200

800

1400

2000

MEAN

200

800

MEAN

1430

2030

MEAN

230

1500

2100

MEAN

300
900
1500
2100

MEAN

$$
\begin{array}{r}
300 \\
900 \\
1500 \\
2100
\end{array}
$$

MEAN

\section{TSS}

$(M G / L)$.

20

8

14

3

9

7

7

7

10

11

12

12

11

20

23

21

13

7

10

23

13

12

16

18

1.4

10

8

12

14

16

16

12

14

\section{FLOW}

(CFS)

70

68

69

68

68

68

68

68

66

66

68

68

67

72

83

78

84

83

84

81

76

76

78

76

76

67

76

74

76

74

74

73

74 
APPENDIX TABLE A1. (CONT.)

\section{DATE}

$12 / 14 / 90$

$12 / 14 / 90$

$12 / 14 / 90$

$12 / 14 / 90$

$12 / 14 / 90$

$12 / 15 / 90$

$12 / 15 / 90$

$12 / 15 / 90$

$12 / 15 / 90$

$12 / 15 / 90$

$12 / 16 / 90$

$12 / 16 / 90$

$12 / 16 / 90$

$12 / 16 / 90$

$12 / 16 / 90$

$12 / 18 / 90$

$12 / 18 / 90$

$12 / 18 / 90$

$12 / 18 / 90$

$12 / 19 / 90$

$12 / 19 / 90$

$12 / 19 / 90$

$12 / 19 / 90$

$12 / 19 / 90$

$12 / 20 / 90$

$12 / 20 / 90$

$12 / 20 / 90$

$12 / 20 / 90$

$12 / 20 / 90$

$12 / 21 / 90$

$12 / 21 / 90$

$12 / 21 / 90$

$12 / 21 / 90$

$12 / 21 / 90$
TIME

300

900

1500

2100

MEAN

300

900

1500

2100

MEAN

300

900

1500

2100

MEAN

1000

1600

2200

MEAN

400

1000

1600

2200

MEAN

400

1000

1600

2200

MEAN

400

1000

1600

2200

MEAN
TS8

$(M G / L)$

14

19

15

14

15

19

9

13

15

14

12

7

13

16

12

14

11

17

14

24

21

17

25

21

41

36

23

30

32

32

24

14

44

28
FLOW

(CFB)

74

78

78

78

77

78

77

77

76

77

76

75

76

74

76

89

90

90

90

93

96

94

85

92

84

89

100

97

93

96

94

92

83

91 
APPENDIX TABLE A1. (CONT.)

\section{DATE}

$12 / 22 / 90$

$12 / 22 / 90$

$12 / 22 / 90$

$12 / 22 / 90$

$12 / 22 / 90$

$12 / 23 / 90$

$12 / 23 / 90$

$12 / 23 / 90$

$12 / 23 / 90$

$12 / 90$

$1 / 22 / 91$

$1 / 22 / 91$

$1 / 22 / 91$

$1 / 22 / 91$

$1 / 23 / 91$

$1 / 23 / 91$

$1 / 23 / 91$

$1 / 23 / 91$

$1 / 23 / 91$

$1 / 24 / 91$

$1 / 24 / 91$

$1 / 24 / 91$

$1 / 24 / 91$

$1 / 24 / 91$

$1 / 25 / 91$

$1 / 25 / 91$

$1 / 25 / 91$

$1 / 25 / 91$

$1 / 25 / 91$

$1 / 26 / 91$

$1 / 26 / 91$

$1 / 26 / 91$

$1 / 26 / 91$

$1 / 26 / 91$

\section{TIME}

400

1000

1600

2200

MEAN

400

1000

1600

MEAN

AVE

1100

1700

2300

MEAN

500

1100

1700

2300

MEAN

500

1100

1700

2300

MEAN

500

1100

1700

2300

MEAN

500

1100

1700

2300

MEAN

\section{T88}

(MG/L)

FLOW

(CFB)

24

28

36

22

27

17

23

31

23

17

82

79

78

78

80

77

76

74

76

79

$\begin{array}{rr}9 & 94 \\ 4 & 92 \\ 14 & 92\end{array}$

9

93

82

88

89

93

88

83

101

190

215

147

13

178

145

124

113

140

113

107

104

101

106 
AFPENDIX TABLE A1. (CONT.)

\section{DATE}

$1 / 27 / 91$

$1 / 27 / 91$

$1 / 27 / 91$

$1 / 27 / 91$

$1 / 27 / 91$

$1 / 29 / 91$

$1 / 29 / 91$

$1 / 29 / 91$

$1 / 29 / 91$

$1 / 30 / 91$

$1 / 30 / 91$

$1 / 30 / 91$

$1 / 30 / 91$

$1 / 30 / 91$

$1 / 31 / 91$

$1 / 31 / 91$

$1 / 31 / 91$

$1 / 31 / 91$

$1 / 31 / 91$

$01 / 91$

$2 / 1 / 91$

$2 / 1 / 91$

$2 / 1 / 91$

$2 / 1 / 91$

$2 / 1 / 91$

$2 / 2 / 91$

$2 / 2 / 91$

$2 / 2 / 91$

$2 / 2 / 91$

$2 / 2 / 91$

$2 / 3 / 91$

$2 / 3 / 91$

$2 / 3 / 91$

$2 / 3 / 91$

\section{TIME}

500

11.00

1700

2300

MEAN

1100

1.700

2300

MEAN

500

1100

1700

2300

MEAN

500

1100

1700

2300

MEAN

AVE

500

1100

1700

2300

MEAN

500
1100
1700
2300

MEAN

500

1100

1700

2300 $\begin{array}{ll}\text { TSS } & \text { FLOW } \\ \text { (MG L L } & \text { (CFS) }\end{array}$

$5 \quad 101$

101

99

99

100

97

97

117

$12 \quad 104$

$18 \quad 104$

$39 \quad 282$

$52 \quad 600$

$27 \quad 435$

$34 \quad 355$

$18 \quad 300$

$15 \quad 202$

12163

$15 \quad 132$

$15 \quad 199$

$13 \quad 153$

$16 \quad 137$

$14 \quad 125$

$18 \quad 122$

$10 \quad 108$

$14 \quad 123$

$13 \quad 108$

$11 \quad 111$

$13 \quad 106$

$14 \quad 106$

$13 \quad 108$

$15 \quad 108$

$16 \quad 1.03$

$6 \quad 103$

$6 \quad 103$ 
APPENDIX TABLE Al. (CONT,)

DATE

$2 / 3 / 91$

$2 / 4 / 91$

$2 / 5 / 91$

$2 / 5 / 91$

$2 / 5 / 91$.

$2 / 5 / 91$

$2 / 5 / 91$

$2 / 6 / 91$

$2 / 6 / 91$

$2 / 6 / 91$

$2 / 6 / 91$

$2 / 6 / 91$

$2 / 6 / 91$

$2 / 6 / 91$

$2 / 6 / 91$

$2 / 6 / 91$

$2 / 6 / 91$

$2 / 6 / 91$.

$2 / 6 / 91$

$2 / 6 / 91$

$2 / 7 / 91$

$2 / 7 / 91$

$2 / 7 / 91$

$2 / 7 / 91$.

$2 / 7 / 91$

$2 / 7 / 91$

$2 / 7 / 91$

$2 / 7 / 91$

$2 / 7 / 91$

$2 / 7 / 91$

$2 / 7 / 91$

$2 / 7 / 9.1$

$2 / 7 / 91$

$2 / 8 / 91$

$2 / 8 / 91$

$2 / 8 / 91$

$2 / 8 / 91$

$2 / 8 / 91$

$2 / 8 / 91$

$2 / 8 / 91$

\section{TIME}

MEAN

500

1800

2000

2200

2400

MEAN

200

400

600

800

1000

1200

1400

1600

1800

2000

2200

2400

MEAN

200

400

600

800

1000

1200

1400

1600

1800

2000

2200

2400

MEAN

200

400

600

800

1000

1200

1400
Ts8

$(M G / L)$

11

9

7

9

13

6

8

8

10

8

5

7

9

3

8

11

15

29

30

12

31

37

36

29

14

2

1

22

7

19

14

12

18

1.5

18

15

12

14

1

14
FLOW

(CFB)

104

101

97

97

97

97

97

94

94

94

94

94

94

97

106

111

106

154

154

108

129

159

198

242

265

300

319

254

354

254

325

297

258

288

271

252

248

237

226

215 
APPENDIX TABLE A. (CONT.)

\section{DATE}

$2 / 8 / 91$

$2 / 8 / 91$

$2 / 8 / 91$

$2 / 8 / 91$

$2 / 8 / 91$

$2 / 8 / 91$

$2 / 9 / 91$

$2 / 9 / 91$

$2 / 9 / 91$

$2 / 9 / 91$.

$2 / 9 / 91$

$2 / 9 / 91$

$2 / 9 / 91$

$2 / 9 / 91$

$2 / 9 / 91$

$2 / 9 / 91$

$2 / 9 / 91$

$2 / 9 / 91$

$2 / 9 / 91$

$2 / 10 / 91$

$2 / 10 / 91$

$2 / 10 / 91$

$2 / 10 / 91$

$2 / 10 / 91$

$2 / 10 / 91$

$2 / 11 / 91$

$2 / 11 / 91$

$2 / 11 / 91$

$2 / 11 / 91$

$2 / 11 / 91$

$2 / 11 / 91$

$2 / 11 / 91$

$2 / 11 / 91$

$2 / 11 / 91$

$2 / 12 / 91$

$2 / 12 / 91$

$2 / 12 / 91$

$2 / 12 / 91$

$2 / 12 / 91$
TIME

1600

1800

2000

2200

2400

MEAN

200

400

600

800

930

1130

1330

1530

1730

1930

2130

2330

MEAN

130

330

530

730

930

MEAN

930

1130

1330

1530

1730

1930

2130

2230

MEAN

130

330

530

730

930
TS8

(MG/L)

13

13

15

14

14

13

14

12

12

14

12

8
19

19

7

6

10

8
20

.11

25

25

38

28

109

45

58

66

68

50

58

52

49

45

56

32

77

38

77

50
FLOW

(CFB)

210

206

206

202

198

231

206

204

190

206

206

196

192

174

198

200

218

248

203

231

259

277

291

306

273

457

500

532

549

549

543

538

543

527

538

158

107

240

210 
APPENDIX TABLE A1. (CONT.)

\section{DATE}

$2 / 12 / 91$

$2 / 12 / 91$

$2 / 12 / 91$

$2 / 12 / 91$

$2 / 12 / 91$

$2 / 12 / 91$

$2 / 12 / 91$

$2 / 12 / 91$

$2 / 13 / 91$

$2 / 13 / 91$

$2 / 13 / 91$

$2 / 13 / 91$

$2 / 13 / 91$.

$2 / 13 / 91$

$2 / 13 / 91$

$2 / 13 / 91$

$2 / 14 / 91$

$2 / 14 / 91$

$2 / 14 / 91$

$2 / 14 / 91$

$2 / 14 / 91$

$2 / 14 / 91$

$2 / 14 / 91$

$2 / 14 / 91$

$2 / 14 / 91$

$2 / 14 / 91$

$2 / 14 / 91$.

$2 / 14 / 91$

$2 / 14 / 91$

$2 / 14 / 91$

$2 / 15 / 91$

$2 / 15 / 91$

$2 / 15 / 91$

$2 / 15 / 91$

$2 / 15 / 91$

$2 / 15 / 91$

$2 / 15 / 91$

$2 / 15 / 91$

$2 / 15 / 91$

$2 / 15 / 91$

$2 / 15 / 91$

$2 / 15 / 91$
TIME

1130

1330

1530

1730

1930

2130

2330

MEAN

130

330

530

730

1000

1600

2200

MEAN

400

1000

1400

1500

1600

1700

1800

1900

2000

2.100

2200

2300

2400

MEAN

100

200

300

400

500

600

700

800

900

930

1130

1330
T88 FLOW

$(M G / L)$ (CFB)

$43 \quad 369$

$35 \quad 511$

$27 \quad 560$

$31 \quad 577$

$27 \quad 242$

$29 \quad 577$

$24 \quad 435$

$41 \quad 377$

$25 \quad 176$

$24 \quad 134$

$43 \quad 237$

$40 \quad 306$

$31 \quad 511$

$26 \quad 543$

$25 \quad 522$

$30 \quad 347$

$23 \quad 511$

$26 \quad 516$

$21 \quad 506$

$19 \quad 506$

$18 \quad 495$

19495

$17 \quad 481$

$17 \quad 476$

$20 \quad 404$

$23 \quad 350$

$32 \quad 396$

$24 \quad 448$

$23 \quad 422$

$21 \quad 462$

$20 \quad 388$

$23 \quad 339$

$23 \quad 354$

$21 \quad 392$

$19 \quad 417$

$17 \quad 435$

$17 \quad 435$

$19 \quad 435$

$19 \quad 426$

$27 \quad 430$

$25 \quad 430$

$25 \quad 430$ 
APPENDIX TABLE AI. (CONT.)

\section{DATE}

$2 / 15 / 91$

$2 / 15 / 91$

$2 / 15 / 91$

$2 / 15 / 91$

$2 / 15 / 91$

$2 / 19 / 91$

$2 / 19 / 91$

$2 / 19 / 91$

$2 / 19 / 91$

$2 / 19 / 91$

$2 / 19 / 91$

$2 / 19 / 91$

$2 / 19 / 91$

$2 / 19 / 91$

$2 / 20 / 91$

$2 / 20 / 91$

$2 / 20 / 91$

$2 / 20 / 91$

$2 / 20 / 91$

$2 / 20 / 91$

$2 / 20 / 91$

$2 / 20 / 91$

$2 / 20 / 91$

$2 / 20 / 91$.

$2 / 20 / 91$

$2 / 20 / 91$

$2 / 20 / 91$

$2 / 21 / 91$

$2 / 21 / 91$

$2 / 21 / 91$

$2 / 21 / 91$

$2 / 21 / 91$

$2 / 21 / 91$.

$2 / 21 / 91$

$2 / 21 / 91$

$2 / 21 / 91$

$2 / 21 / 91$

$2 / 21 / 91$

$2 / 21 / 91$

$2 / 21 / 91$
TIME

1530

1730

1930

2130

MEAN

1000

1200

1400

1600

1800

2000

2200

2400

MEAN

200

400

600

800

1000

1200

1400

1600

1800

2000

2200

2400

MEAN

200

400

600

800

1000

1200

1400

1600

1800

2000

22.00

2400

MEAN
T88

$(M G / L)$

24

22

19

18

21

7

7

10

15

8

11

1.8

7

10

24

20

22

19

1.4

14

15

20

14

1. 1

17

14

17

9

9

1

8

10

8

9

9

5

8

9

5

7
FLOW

(CF8).

422

422

422

422

412

158

161

161

147

108

128

120

120

138

129

186

184

186

182

182

186

182

182

184

182

188

179

168

165

165

158

151

.247

137

144

140

13.1

128

125

146 
APPENDIX TABLE AI. (CONT. )

\section{DATE}

$2 / 22 / 91$

$2 / 22 / 91$

$2 / 22 / 91$

$2 / 22 / 91$

$2 / 22 / 91$

$2 / 22 / 91$

$2 / 22 / 91$

$2 / 22 / 91$

$2 / 23 / 91$

$2 / 23 / 9.1$

$2 / 23 / 91$

$2 / 23 / 91$

$2 / 23 / 91$

$2 / 24 / 91$

$2 / 24 / 91$

$2 / 24 / 91$

$2 / 24 / 91$

$2 / 24 / 91$

$2 / 25 / 91$

$2 / 25 / 91$

$2 / 25 / 91$

$2 / 25 / 91$

$2 / 25 / 91$

$2 / 26 / 91$

$2 / 26 / 91$

$2 / 26 / 91$

$2 / 26 / 91$

$2 / 26 / 91$

$2 / 27 / 91$

$2 / 27 / 91$

$2 / 27 / 91$

$2 / 27 / 91$

$2 / 27 / 91$

$2 / 28 / 91$

$02 / 91$
TIME

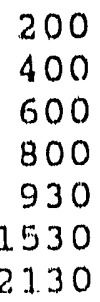

MEAN

330

930

1530

2130

MEAN

330

930

1530

2130

MEAN

330

930

1530

2130

MEAN

330

930

1530

2130

MEAN

330

930

1530

2130

MEAN

330

MEAN
TSB FLOW

$(M G / L) \quad$ (CFB)

$\begin{array}{rr}8 & 132 \\ 9 & 125 \\ 8 & 117 \\ 15 & 140 \\ 6 & 128 \\ 7 & 122 \\ 8 & 128\end{array}$

127

131

129

123

122

126

121

106

103

100

$11 \quad 107$

$20 \quad 159$

$16 \quad 168$

$16 \quad 158$

$16 \quad 2 ! 6$

$17 \quad 160$

$23 \quad 122$

1296

1093

$9 \quad 93$

$13 \quad 101$

$10 \quad 94$

93

94

88

92

83

$19 \quad 242$ 
APPENDIX TABLE AL. (CONI'.)

DATE

$3 / 5 / 91$

$3 / 5 / 91$

$3 / 5 / 91$

$3 / 5 / 91$

$3 / 6 / 91$

$3 / 6 / 91$

$3 / 6 / 91$.

$3 / 6 / 91$

$3 / 6 / 91$

$3 / 7 / 91$

$3 / 7 / 91$

$3 / 7 / 91$

$3 / 7 / 91$

$3 / 7 / 91$

$3 / 8 / 91$

$3 / 8 / 91$

$3 / 8 / 91$

$3 / 8 / 91$

$3 / 8 / 91$

$3 / 9 / 91$.

$3 / 9 / 91$

$3 / 9 / 91$

$3 / 9 / 91$

$3 / 9 / 91$

$3 / 10 / 91$

$3 / 10 / 91$

$3 / 10 / 91$

$3 / 10 / 91$

$3 / 10 / 91$

$3 / 11 / 91$

$3 / 12 / 91$

$3 / 12 / 91$.

$3 / 12 / 91$

$3 / 12 / 91$
TIME

900

1500

21.00

MEAN

300

900

1500

2100

MEAN

300

900

1500

2100

MEAN

300

900

1500

2200

MEAN

300

900

1500

2.100

MEAN

300

900

1500

2.100

MEAN

300

1000

1600

2.200

MEAN
TS8 FLOW

$(M G / L L$ (CFB)

26

$38 \quad 350$

24

350

$29 \quad 353$

$24 \quad 322$

$1.4 \quad 373$

$36 \quad 549$

$24 \quad 538$

$24 \quad 445$

$18 \quad 413$

$27 \quad 404$

$20 \quad 298$

$28 \quad 409$

$23 \quad 381$

$26 \quad 404$

$17 \quad 422$

$15 \quad 329$

$20 \quad 268$

$19 \quad 356$

$16 \quad 245$

$15 \quad 251$

$14 \quad 242$

4245

$12 \quad 246$

$9 \quad 481$

$20 \quad 202$

$12 \quad 188$

$14 \quad 194$

$14 \quad 266$

$12 \quad 176$

$10 \quad 245$

$17 \quad 192$

$18 \quad 292$

$15 \quad 210$ 
APPENDIX TABLE A. . (CON'T.)

\section{DATE}

$3 / 13 / 91$

$3 / 13 / 91$

$3 / 13 / 91$

$3 / 1.3 / 91$

$3 / 13 / 91$

$3 / 14 / 91$

$3 / 14 / 91$

$3 / 14 / 91$

$3 / 14 / 91$

$3 / 14 / 91$

$3 / 15 / 91$

$3 / 15 / 91$

$3 / 15 / 91$

$3 / 15 / 91$

$3 / 15 / 91$

$3 / 16 / 91$

$3 / 16 / 91$

$3 / 16 / 91$

$3 / 16 / 91$

$3 / 26 / 91$

$3 / 17 / 91$

$3 / 17 / 91$

$3 / 17 / 91$

$3 / 17 / 91$

$3 / 17 / 91$

$3 / 18 / 91$

$3 / 19 / 91$

$3 / 19 / 91$

$3 / 19 / 91$

$3 / 19 / 91$

$3 / 20 / 91$

$3 / 20 / 91$

$3 / 20 / 91$

$3 / 20 / 91$
IIME

400

1000

1600

2200

MEAN

400

1000

1600

2200

MEAN

400

1000

1600

2200

MEAN

400

$10 \cap 0$

1600

2200

MEAN

400

1000

1600

2200

MEAN

400

1000

1600

2200

MEAN

400

1000

1600

2200
TS8 FLOW

$(M G / L)$ (CFB)

$24 \quad 168$

$18 \quad 184$

$19 \quad 229$

12280

$.28 \quad 215$

7245

$14 \quad 476$

$12 \quad 457$

$12 \quad 138$

$11 \quad 329$

11200

74

234

231

185

206

229

226

210

218

127

259

256

256

225

417

430

457

471

$21 \quad 453$

$20 \quad 248$

$16 \quad 384$

$16 \quad 392$

14384 
APPENDTX TABLE A1. (CONT.)

\section{DATE}

$3 / 20 / 91$

$3 / 21 / 91$

$3 / 21 / 91$

$3 / 21 / 91$

$3 / 21 / 91$

$3 / 21 / 91$

$3 / 22 / 91$

$3 / 22 / 91$

$3 / 22 / 91$

$3 / 22 / 91$

$3 / 22 / 91$

$3 / 23 / 91$

$3 / 23 / 91$

$3 / 23 / 91$

$3 / 23 / 91$

$3 / 23 / 91$

$3 / 24 / 91$

$3 / 24 / 91$

$3 / 24 / 91$.

$3 / 24 / 91$

$3 / 24 / 91$

$3 / 25 / 91$

$3 / 26 / 91$

$3 / 26 / 91$

$3 / 26 / 91$

$3 / 26 / 91$

$3 / 27 / 91$

$3 / 27 / 91$

$3 / 27 / 91$

$3 / 27 / 91$

$3 / 27 / 91$

$3 / 28 / 91$

$3 / 28 / 91$

$3 / 28 / 91$
TIME

MEAN

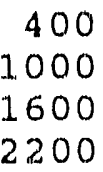

MEAN

400

1000

1600

2200

MEAN

400

1000

1600

2200

MEAN

400

1000

1600

2200

MEAN

400

930

1530

2130

MEAN

330

930

1530

2130

MEAN

330

930

1530
Ts8

(MG/L)

16

19

23

14

14

17

19

13

10

13

13

11

19

16

16

15

12

1.4

11

11

12

19

7

16

59

27

37

18

28

22

26

17

17

14
FLOW

(CFB)

352

388

34.3

377

381

372

404

396

396

396

398

396

1.72

137

49

188

70

76

74

68

72

67

138

117

481

245

369

358

313

381.

355

377

373

377 
APPENDIX TABLE AI. (CONT.)

DATE

$3 / 28 / 91$

$3 / 28 / 91$

$3 / 29 / 91$

$3 / 29 / 91$

$3 / 29 / 91$

$3 / 29 / 91$

$3 / 29 / 91$

$3 / 30 / 91$

$3 / 30 / 91$

$3 / 30 / 91$

$3 / 30 / 91$.

$3 / 30 / 91$

$3 / 31 / 91$

$3 / 31 / 91$

$3 / 31 / 91$

$3 / 31 / 91$

$3 / 31 / 91$

$03 / 91$

$4 / 1 / 91$

$4 / 1 / 91$

$4 / 1 / 91$

$4 / 1 / 91$

$4 / 2 / 91$

$4 / 2 / 91$

$4 / 2 / 91$

$4 / 2 / 91$

$4 / 2 / 91$

$4 / 2 / 91$

$4 / 3 / 91$

$4 / 3 / 91$

$4 / 3 / 91$

$4 / 3 / 91$

$4 / 3 / 91$
TIME

2130

MEAN

330

930

1530

2130

MEAN

330

930

1530

2130

MEAN

330

930

1530

2130

MEAN

AVE

330

1030

1630

2230

MEAN

430

1030

1630

2230

MEAN

430

1030

1630

2230

MEAN
TSS FLOW

$(M G / L) \quad$ (CFS)

17

373

$16 \quad 375$

$15 \quad 506$

$15 \quad 168$

$46 \quad 56$

$28 \quad 358$

$26 \quad 272$

$20 \quad 329$

$17 \quad 291$

$15 \quad 161$

$11 \quad 122$

$16 \quad 226$

$18 \quad 154$

$14 \quad 140$

$12 \quad 132$

$19 \quad 168$

$16 \quad 149$

$17 \quad 284$

$17 \quad 144$

1193

$15 \quad 12.5$

$18 \quad 131$

$15 \quad 123$

$21 \quad 129$

$12 \quad 127$

$14 \quad 131$

$17 \quad 132$

$16 \quad 130$

$22 \quad 131$

$22 \quad 129$

$22 \quad 128$

$23 \quad 127$

$22 \quad 129$ 
APPENDIX TABLE A1. (CONT.)

\section{DATE}

$4 / 4 / 91$

$4 / 4 / 91$

$4 / 4 / 91$

$4 / 4 / 91$

$4 / 4 / 91$

$4 / 5 / 91$

$4 / 5 / 91$

$4 / 5 / 91$

$4 / 5 / 91$

$4 / 5 / 91$

$4 / 6 / 91$

$4 / 6 / 91$

$4 / 6 / 91$

$4 / 8 / 91$

$4 / 8 / 91$

$4 / 8 / 91$

$4 / 8 / 91$

$4 / 9 / 91$

$4 / 9 / 91$

$4 / 9 / 91$

$4 / 9 / 91$

$4 / 9 / 91$

$4 / 10 / 91$

$4 / 10 / 91$

$4 / 10 / 91$

$4 / 10 / 91$

$4 / 10 / 91$

$4 / 11 / 91$

$4 / 11 / 91$

$4 / 11 / 91$

$4 / 11 / 91$

$4 / 11 / 91$

$4 / 12 / 91$

$4 / 12 / 91$
TIME

430

1030

1630

2230

MEAN

430

1030

1630

2230

MEAN

430

1030

MEAN

1000

1600

2200

MEAN

400

1000

1600

2200

MEAN

400

1000

1600

2200

MEAN

400

1000

1600

2200

MEAN

400

1000

\section{T88}

(MG/L)

19

16

16

14

16

22

14

34

22

23

24

16

20

11

10

10

10

14

11

14

7

11

12

13

14

11

12

9

10

12

11

10

12

16
FLOW

(CFS)

127

127

127

124

126

121

122

231

259

183

259

200

229

144

147

151

147

161

163

132

144

150

137

137

149

142

141

138

121

121

1.28

127

96

104 
APPENDIX TABLE AI. (CONT.)

DATE

$4 / 12 / 91$

$4 / 12 / 91$

$4 / 12 / 91$

$4 / 13 / 91$

$4 / 13 / 91$

$4 / 13 / 91$

$4 / 13 / 91$

$4 / 13 / 91$

$4 / 14 / 91$

$4 / 16 / 91$

$4 / 16 / 91$

$4 / 16 / 91$

$4 / 16 / 91$

$4 / 17 / 91$

$4 / 17 / 91$

$4 / 17 / 91$

$4 / 17 / 91$

$4 / 17 / 91$

$4 / 18 / 91$

$4 / 18 / 91$

$4 / 18 / 91$

$4 / 18 / 91$

$4 / 18 / 91$

$4 / 19 / 91$

$4 / 19 / 91$

$4 / 19 / 91$

$4 / 19 / 91$

$4 / 19 / 91$.

$4 / 20 / 91$

$4 / 20 / 91$

$4 / 20 / 91$

$4 / 20 / 91$

$4 / 20 / 91$
TIME

1600

2200

MEAN

400

1000

2600

2200

MEAN

400

1000

1600

2200

MEAN

400

1000

1600

2200

MEAN

400

1000

1600

2200

MEAN

400

1000

1600

2200

MEAN

400

1000

1600

2200

MEAN
TS8

(MG/L)

11

11

12

43

20

11

10

21

11

9

6

17

11

7

11

12

31.

15

14

10

15

8

12

8

9

8

10

9

6

6

15

8

8
FLON

(CFB)

118

400

180

163

152

121

121

139

124

124

129

118

124

62

122

297

282

191

66

400

444

453

341

204

404

409

413

357

490

485

103

196

318 
APPENDIX TABLE A1. (CONT.)

\section{DATE}

$4 / 21 / 91$

$4 / 21 / 91$

$4 / 21 / 91$

$4 / 21 / 91$

$4 / 21 / 91$

$4 / 22 / 91$

$4 / 23 / 91$

$4 / 23 / 91$

$4 / 23 / 91$

$4 / 23 / 91$

$4 / 24 / 91$

$4 / 24 / 91$

$4 / 24 / 91$

$4 / 24 / 91$

$4 / 24 / 91$

$4 / 25 / 91$

$4 / 25 / 91$

$4 / 25 / 91$

$4 / 25 / 91$

$4 / 25 / 91$

$4 / 26 / 91$

$4 / 26 / 9$ ?

$4 / 26 / 91$

$4 / 26 / 91$

$4 / 26 / 91$

$4 / 27 / 91$

$4 / 27 / 91$

$4 / 27 / 91$

$4 / 27 / 91$

$4 / 27 / 91$

$4 / 28 / 91$

$4 / 28 / 91$

$4 / 28 / 91$

$4 / 28 / 91$
TIME

400

1000

1600

2200

MEAN

400

900

1500

2100

MEAN

300

900

1500

2100

MEAN

300

900

1500

2100

MEAN

300

900

1.500

2100

MEAN

300

900

1500

2100

MEAN

300

900

1500

2100
T88

(MG/L)

$\begin{array}{ll}5 & 381 \\ 9 & 381 \\ 9 & 381 \\ 7 & 373 \\ 8 & 379 \\ 12 & 373 \\ 16 & 373 \\ 13 & 310 \\ 11 & 373\end{array}$

13

357

10

1. 1

18

13

13

8

4
12

9

8

8

8

9

8

8

64

4
48

52

64

138

145

100

159

122

310

285 
APPENDIX TABLE A.I, (CONT.)

\section{DATE}

$4 / 28 / 91$

$4 / 29 / 91$

$4 / 30 / 91$

$4 / 30 / 91$

$4 / 30 / 91$

$04 / 91$

$5 / 1 / 91$

$5 / 1 / 91$

$5 / 1 / 91$

$5 / 1 / 91$

$5 / 1 / 91$

$5 / 2 / 91$

$5 / 2 / 91$

$5 / 2 / 91$

$5 / 2 / 91$

$5 / 2 / 91$

$5 / 3 / 91$

$5 / 3 / 91$

$5 / 3 / 91$

$5 / 3 / 91$

$5 / 3 / 91$

$5 / 4 / 91$

$5 / 4 / 91$

$5 / 4 / 91$

$5 / 4 / 91$

$5 / 4 / 91$

$5 / 5 / 91$

$5 / 5 / 91$

$5 / 5 / 91$

$5 / 5 / 91$

$5 / 5 / 91$

$5 / 7 / 91$

$5 / 7 / 91$
TIME

MEAN

300

1500

2100

MEAN

AVE

300

900

1500

2100

MEAN

300

900

1500

2100

MEAN

300

900

1500

2100

MEAN

300

900

1500

2100

MEAN

300

900

1500

2100

MEAN

800

1400

\section{TSS FIOW}

(MG/L) (CFE).

$13 \quad 219$

13208

$16 \quad 322$

1493

$15 \quad 207$

$11 \quad 196$

1197

1292

$13 \quad 106$

$10 \quad 110$

$11 \quad 101$

$11 \quad 97$

79

76

75

82

73

137

137

140

122

1.17

117

120

128

120

134

134

147

14251

$13 \quad 166$

$10 \quad 152$

128 
APPENDIX TABLE A1. (CONT.)

\section{DATE}

$5 / 7 / 91$

$5 / 7 / 91$

$5 / 8 / 91$

$5 / 8 / 91$

$5 / 8 / 91$

$5 / 8 / 91$

$5 / 8 / 91$

$5 / 9 / 91$

$5 / 9 / 91$

$5 / 9 / 91$

$5 / 9 / 91$

$5 / 9 / 91$

$5 / 10 / 91$

$5 / 10 / 91$

$5 / 10 / 91$

$5 / 10 / 91$

$5 / 10 / 91$

$5 / 11 / 91$

$5 / 11 / 91$

$5 / 11 / 91$

$5 / 11 / 91$

$5 / 11 / 91$

$5 / 12 / 91$

$5 / 12 / 91$

$5 / 12 / 91$

$5 / 12 / 91$

$5 / 12 / 91$

$5 / 13 / 91$

$5 / 14 / 91$

$5 / 14 / 91$

$5 / 14 / 91$

$5 / 14 / 91$

$5 / 15 / 91$
TIME

2000

MEAN

200

800

1400

2000

MEAN

200

800

1400

2000

MEAN

200

800

1400

2000

MEAN

200

800

1400

2000

MEAN

200

800

1400

2000

MEAN

200

800

1400

2000

MEAN

200
Ts8

$(M G / L)$

6

7

6

6

5

10

7

11

12

9

11

1.1

10

9

6

8

8

11

8

6

8

8

10

7

10

12

10

7

19

17

16

17

12
FLOW

(CFB)

77

119

72

69

79

70

73

69

64

62

73

67

73

71

68

63

69

63

63

62

60

62

59

59

59

54

58

63

93

83

72

83

68 
APPENDIX TABLE A1. (CON'T'.)

\section{DATE}

$5 / 15 / 91$.

$5 / 15 / 91$

$5 / 15 / 91$

$5 / 15 / 91$

$5 / 16 / 91$

$5 / 16 / 91$

$5 / 16 / 91$

$5 / 16 / 91$

$5 / 16 / 91$

$5 / 17 / 91$

$5 / 17 / 91$

$5 / 17 / 91$

$5 / 17 / 91$

$5 / 17 / 91$

$5 / 18 / 91$

$5 / 18 / 91$

$5 / 18 / 91$

$5 / 18 / 91$

$5 / 18 / 91$

$5 / 21 / 91$

$5 / 21 / 91$

$5 / 21 / 91$

$5 / 21 / 91$

$5 / 22 / 91$

$5 / 22 / 91$

$5 / 22 / 91$

$5 / 22 / 91$

$5 / 22 / 91$

$5 / 23 / 91$

$5 / 23 / 91$

$5 / 23 / 91$

$5 / 23 / 91$

$5 / 23 / 91$

$5 / 24 / 91$

\section{TIME}

800

1400

2000

MEAN

200

800

1400

2000

MEAN

$$
200
$$

800

1400

2000

MEAN

200

800

1400

2000

MEAN

800
1400
2000

MEAN

$$
\begin{array}{r}
200 \\
800 \\
1400 \\
2000
\end{array}
$$

MEAN

200

800

1400

2000

MEAN

2013
TS8

(MG/L)

13

30

8

16

15

16

26

21

19

23

12

16

25

19

21.

19

21

24

21

46

32

21

33

30

26

16

12

21

23

16

35

20

23

21

40
FLOW

(CFB)

63

59

56

61

56

53

47

46

50

46

46

46

50

47

46

46

45

45

45

52

54

53

53

53

45

45

45

47

45

45

45

45

45

45 


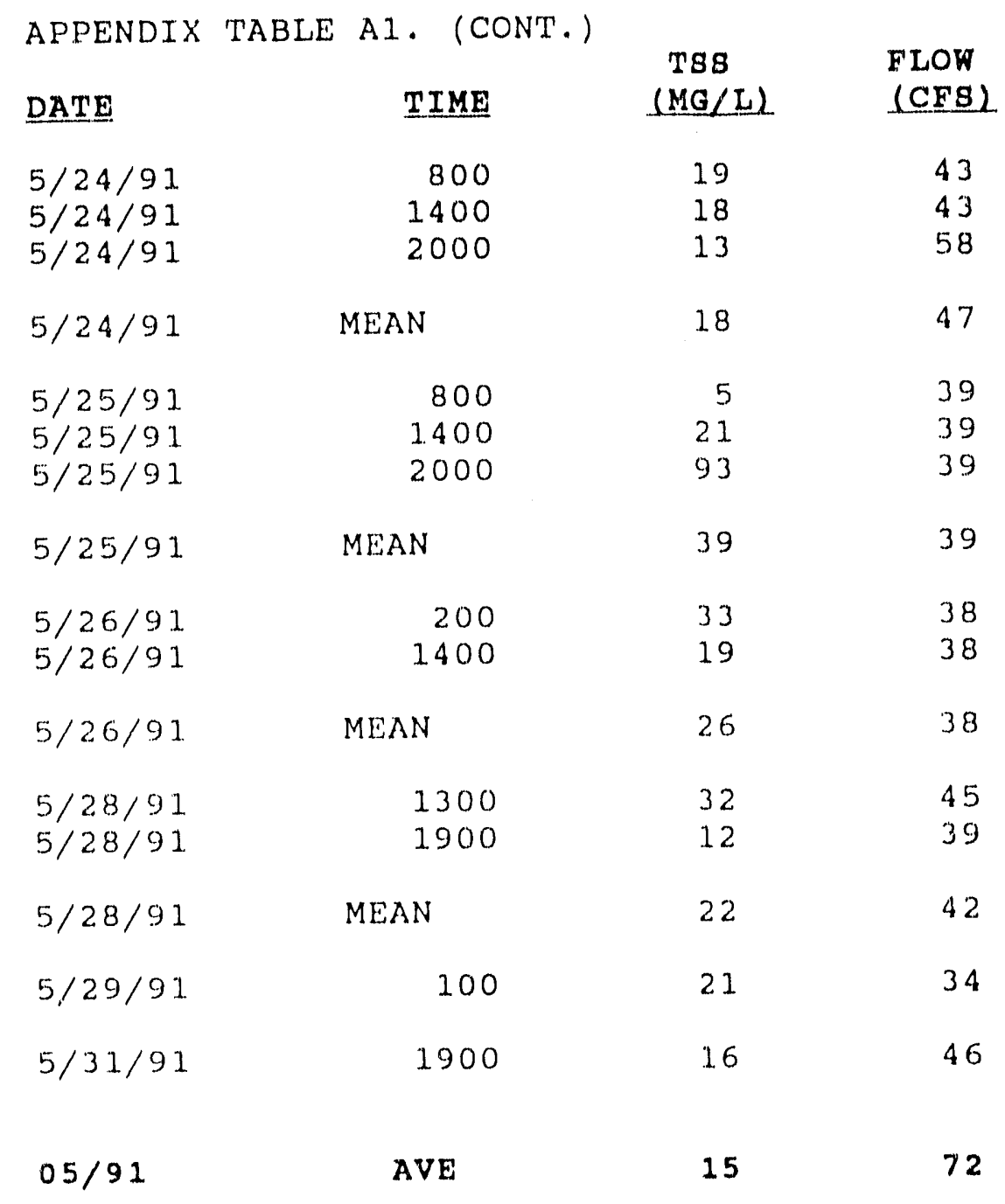


Appendix Table A2. Total suspended solids (TS8) and Flow in Indian Grave Branch at Road B, October 1990 through May 1991.

\begin{tabular}{|c|c|c|c|}
\hline DATE & TIME & $\begin{array}{c}\text { TS8 } \\
(M G / L)\end{array}$ & $\begin{array}{l}\text { FLOW } \\
\text { (CF' } 8 \text { ) }\end{array}$ \\
\hline$\overline{10 / 12 / 90}$ & $\overline{1115}$ & 106 & 52 \\
\hline $10 / 12 / 90$ & 1515 & 21 & 52 \\
\hline $10 / 12 / 90$ & 1915 & 70 & 52 \\
\hline $10 / 12 / 90$ & 2315 & 7 & 52 \\
\hline $10 / 12 / 90$ & MEAN & 51 & 52 \\
\hline $10 / 13 / 90$ & 315 & 5 & 52 \\
\hline $10 / 13 / 90$ & 715 & 60 & 52 \\
\hline $10 / 13 / 90$ & 1115 & 6 & 52 \\
\hline $10 / 13 / 90$ & 1515 & 7 & 52 \\
\hline $10 / 13 / 90$ & 1915 & 18 & 46 \\
\hline $10 / 13 / 90$ & 2315 & 8 & 46 \\
\hline $10 / 13 / 90$ & MEAN & 17 & 50 \\
\hline $10 / 14 / 90$ & 315 & 28 & 46 \\
\hline $10 / 14 / 90$ & 715 & 15 & 48 \\
\hline $10 / 14 / 90$ & 1115 & 14 & 48 \\
\hline $10 / 14 / 90$ & 151.5 & 15 & 46 \\
\hline $10 / 14 / 90$ & 1915 & 18 & 46 \\
\hline $10 / 14 / 90$ & 2315 & 51 & 44 \\
\hline $10 / 1.4 / 90$ & MEAN & 23 & 46 \\
\hline $10 / 15 / 90$ & 315 & 26 & 50 \\
\hline $10 / 15 / 90$ & 715 & 31 & 52 \\
\hline $10 / 25 / 90$ & 1115 & 27 & 52 \\
\hline $10 / 15 / 90$ & 1515 & 22 & 44 \\
\hline $10 / 15 / 90$ & 1915 & 29 & 52 \\
\hline $10 / 15 / 90$ & 2315 & 20 & 56 \\
\hline $10 / 15 / 90$ & MEAN & 26 & 51 \\
\hline $10 / 16 / 90$ & 315 & 26 & 56 \\
\hline $10 / 16 / 90$ & 715 & 23 & 64 \\
\hline $10 / 16 / 90$ & MEAN & 24 & 60 \\
\hline $10 / 90$ & AVE & 27 & 5.1 \\
\hline $11 / 28 / 90$ & 1800 & 4 & 48 \\
\hline \multirow[t]{2}{*}{$11 / 29 / 90$} & 1800 & 4 & 48 \\
\hline & \multicolumn{3}{|c|}{42} \\
\hline
\end{tabular}


APPENDIX TABLE A2 - (CON'I')

\section{DATE}

$11 / 30 / 90$

$11 / 90$

$12 / 01 / 90$

$12 / 02 / 90$

$12 / 03 / 90$

$12 / 04 / 90$

$12 / 04 / 90$

$12 / 04 / 90$

$12 / 05 / 90$

$12 / 10 / 90$

$12 / 11 / 90$

$12 / 11 / 90$

$12 / 11 / 90$

$12 / 12 / 90$

$12 / 12 / 90$

$12 / 12 / 90$

$12 / 12 / 90$

$12 / 12 / 90$

$12 / 13 / 90$

$12 / 13 / 90$

$12 / 13 / 90$

$12 / 13 / 90$

$12 / 13 / 90$

$12 / 14 / 90$

$12 / 14 / 90$

$12 / 14 / 90$

$12 / 14 / 90$

$12 / 14 / 90$

$12 / 15 / 90$

$12 / 15 / 90$
TIME

1800

AVE

1800

1800

1800

1500

2000

MEAN

200

1530

1400

2000

MEAN

200

800

1400

2000

MEAN

200

800

1400

2000

MEAN

200

800

1400

2000

MEAN

200

800
T88

$(M G / L)$.

4

1

53

60

60

60

60

60

60

62

74

69

69

69

69

69

69

69

69

67

64

64

64

65

71

69

69

69

70

69

69 
APPENDIX TABLE A2, (CONT')

\section{DATE}

$12 / 15 / 90$

$12 / 15 / 90$

$12 / 15 / 90$

$12 / 16 / 90$

$12 / 16 / 90$

$12 / 16 / 90$

$12 / 16 / 90$

$12 / 16 / 90$

$12 / 18 / 90$

$12 / 18 / 90$

$12 / 18 / 90$

$12 / 19 / 90$

$12 / 19 / 90$

$12 / 19 / 90$

$12 / 19 / 90$

$12 / 19 / 90$

$1.2 / 20 / 90$

$12 / 20 / 90$

$12 / 20 / 90$

$12 / 20 / 90$

$12 / 20 / 90$

$12 / 21 / 90$

$12 / 21 / 90$

$12 / 21 / 90$

$12 / 21 / 90$

$12 / 21 / 90$

$12 / 22 / 90$

$12 / 22 / 90$

$12 / 22 / 90$

$12 / 22 / 90$

$12 / 22 / 90$
TIME

1400

2000

MEAN

200

800

1.400

2000

MEAN

900

2100

MEAN

300

900

1500

2100

MEAN

300

900

1500

2100

MEAN

300

900

1500

2100

MEAN

300

900

1500

2100

MEAN
T88

(MO/LL

$\begin{array}{ll}8 & 69 \\ 9 & 67\end{array}$

8

4

8

10

12

8

24

5

1.4

7

14

1.5

10

11

1.3

14

1.2

1.9

1.4

12

12

17

1.0

13

1.8

9

18

15
68

FLOW

(CFB)

67

67

67

64

66

79

79

79

79

85

74

74

78

74

85

74

74

77

74

74

69

64

69

64

64

64

64

64 


\begin{tabular}{|c|c|c|c|}
\hline DATE & TIME & $\begin{array}{c}T 88 \\
(M G / L) .\end{array}$ & $\begin{array}{l}\text { FLOW } \\
\text { (CFB) }\end{array}$ \\
\hline $12 / 23 / 90$ & 300 & 15 & 64 \\
\hline $12 / 23 / 90$ & 900 & 10 & 64 \\
\hline $12 / 23 / 90$ & 1500 & 15 & 64 \\
\hline $12 / 23 / 90$ & MEAN & 13 & 64 \\
\hline $1.2 / 90$ & $A \cup D$ & 11 & 69 \\
\hline $1 / 22 / 91$ & 1000 & 4 & 74 \\
\hline $1 / 22 / 91$ & 1600 & 3 & 74 \\
\hline $1 / 22 / 91$ & 2200 & 3 & 74 \\
\hline $1 / 22 / 91$ & MEAN & 3 & 74 \\
\hline $1 / 23 / 91$ & 400 & 5 & 74 \\
\hline $1 / 22 / 91$ & 1000 & 4 & 74 \\
\hline $1 / 23 / 91$ & 1600 & 3 & 74 \\
\hline $1 / 23 / 91$ & 2200 & 6 & 79 \\
\hline $1 / 23 / 91$ & MEAN & 4 & 75 \\
\hline $1 . / 24 / 91$ & 400 & 2 & 79 \\
\hline $1 / 24 / 91$ & 1000 & 4 & 79 \\
\hline $1 / 24 / 91$ & 1600 & 3 & 79 \\
\hline $1 / 24 / 91$ & 2200 & 4 & 76 \\
\hline $1 / 24 / 91$ & MEAN & 3 & 78 \\
\hline $1 / 25 / 91$ & 400 & 6 & 76 \\
\hline $1 / 25 / 91$ & 1000 & 4 & 79 \\
\hline $1 / 25 / 91$ & 1600 & 2 & 79 \\
\hline $1 / 25 / 91$ & 2200 & 4 & 76 \\
\hline $1 / 25 / 91$ & MEAN & 4 & 78 \\
\hline $1 / 26 / 91$ & 400 & 4 & 79 \\
\hline $1 / 26 / 91$ & 1000 & 3 & 79 \\
\hline $1 / 26 / 91$ & 1600 & 4 & 76 \\
\hline $1 / 26 / 91$ & 2200 & 5 & 76 \\
\hline $1 / 26 / 91$ & MEAN & 4 & 78 \\
\hline $1 / 27 / 91$ & 400 & 4 & 76 \\
\hline $1 . / 27 / 91$ & 1000 & 6 & 76 \\
\hline $1 / 27 / 91$ & 1600 & 3 & 76 \\
\hline $1 / 27 / 91$ & 2200 & 4 & 74 \\
\hline $1 / 27 / 91$ & MEAN & 4 & 76 \\
\hline
\end{tabular}




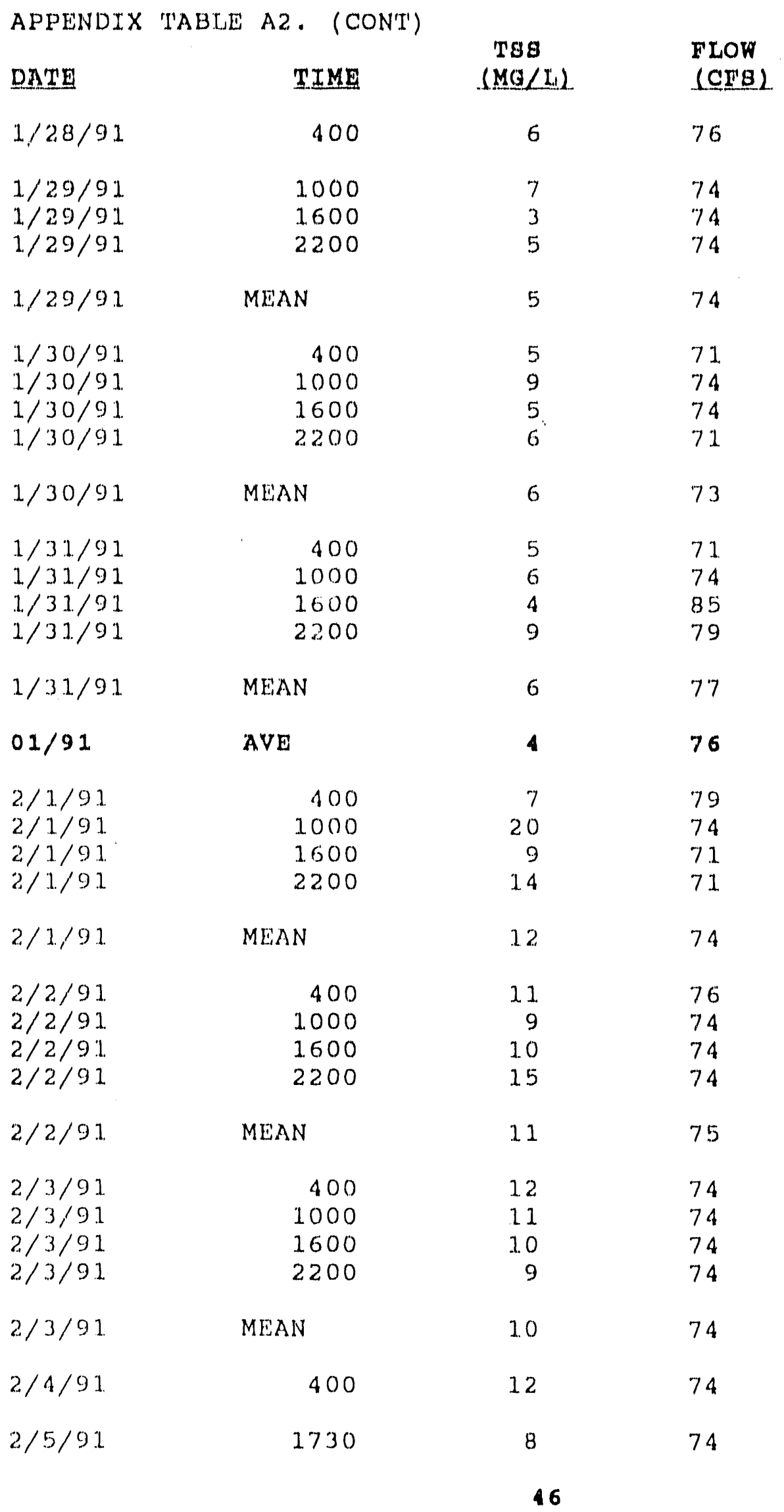


APPENDIX TABLE A2 - (CONT)

\section{DATE}

$2 / 5 / 91$

$2 / 5 / 91$

$2 / 5 / 91$

$2 / 5 / 91$

$2 / 6 / 91$

$2 / 6 / 91$

$2 / 6 / 91$

$2 / 6 / 91$

$2 / 6 / 91$

$2 / 6 / 91$

$2 / 6 / 91$

$2 / 6 / 91$

$2 / 6 / 91$

$2 / 6 / 91$

$2 / 6 / 91$

$2 / 6 / 91$

$\therefore ; 6 / 91$

$2 / 7 / 91$

$2 / 7 / 91$

$2 / 7 / 91$

$2 / 7 / 91$

$2 / 7 / 91$

$2 / 7 / 91$

$2 / 7 / 91$

$2 / 7 / 91$

$2 / 7 / 91$

$2 / 7 / 91$.

$2 / 7 / 91$

$2 / 7 / 91$

$2 / 7 / 91$

$2 / 8 / 91$

$2 / 8 / 91$

$2 / 8 / 91$

$2 / 8 / 91$

$2 / 8 / 91$.

$2 / 8 / 91$

$2 / 8 / 91$

$2 / 8 / 91$

$2 / 8 / 91$

$2 / 8 / 91$

$2 / 8 / 91$

$2 / 8 / 91$
TIME

1930

2.130

2330

MEAN

130

330

530

730

930

1130

1330

1530

1730

1930

2130

2330

MEAN

130

330

530

730

930

1130

1330

1530

1730

1930

2130

2330

MEAN

130

330

530

730

930

1130

1330

1.530

1730

1930

2130

2330
Ts8

$(M G / L L$

$\begin{array}{ll}2 & 74 \\ 6 & 74 \\ 6 & 74\end{array}$

$5 \quad 74$

$5 \quad 74$

474

74

74

75

73

90

85

85

1.27

124

134

91

144

1.44

144

147

150

150

147

150

150

150

150

150

$16 \quad 148$

$17 \quad 150$

$20 \quad 150$

$18 \quad 150$

$20 \quad 150$

$13 \quad 150$

$11 \quad 150$

$13 \quad 149$

$9 \quad 149$

$9 \quad 145$

$11 \quad 144$

$12 \quad 144$

$7 \quad 144$ 
APPENDIX TABLE A2. (CONT)

DATE

$2 / 8 / 91$

$2 / 9 / 91$

$2 / 9 / 91$

$2 / 9 / 91$

$2 / 9 / 91$

$2 / 9 / 91$

$2 / 9 / 91$

$2 / 9 / 91$

$2 / 9 / 91$

$2 / 9 / 91$

$2 / 9 / 91$

$2 / 9 / 91$

$2 / 9 / 91$

$2 / 9 / 91$

$2 / 9 / 91$

$2 / 10 / 91$

$2 / 10 / 91$

$2 / 10 / 91$

$2 / 10 / 91$

$2 / 10 / 91$

$2 / 10 / 91$

$2 / 10 / 91$

$2 / 10 / 91$

$2 / 10 / 91$

$2 / 10 / 91$

$2 / 10 / 91$

$2 / 10 / 91$

$2 / 10 / 91$

$2 / 11 / 91$

$2 / 11 / 91$

$2 / 11 / 91$

$2 / 11 / 91$

$2 / 11 / 91$

$2 / 11 / 91$

$2 / 11 / 91$

$2 / 11 / 91$

$2 / 11 / 91$

$2 / 11 / 91$

$2 / 11 / 91$

$2 / 11 / 91$

$2 / 11 / 91$.
TIME

MEAN

130

330

530

730

845

900

1100

1300

1500

1700

1900

2100

2300

MEAN

100

300

500

700

900

1100

1300

1500

1700

1900

2100

2300

MEAN

100

300

500

700

900

1100

1300

1500

1700

1900

21.00

2300

MEAN
TS8

$(M G / L)$

13

$11 \quad 144$

$15 \quad 144$

12160

$10 \quad 145$

$4 \quad 144$

$8 \quad 144$

$11 \quad 144$

$35 \quad 286$

$11 \quad 158$

$10 \quad 158$

$31 \quad 190$

$26 \quad 198$

$40 \quad 214$

$17 \quad 164$

$116 \quad 240$

$37 \quad 245$

$66 \quad 275$

$71 \quad 270$

$109 \quad 303$

$91 \quad 292$

$67 \quad 292$

$69 \quad 292$

$54 \quad 292$

$54 \quad 303$

$47 \quad 303$

$52 \quad 303$

$69 \quad 284$

$50 \quad 309$

$100 \quad 388$

$116 \quad 400$

$106 \quad 448$

$87 \quad 460$

$133 \quad 466$

$71 \quad 466$

$64 \quad 460$

$50 \quad 460$

$40 \quad 460$

$47 \quad 460$

$48 \quad 214$

$76 \quad 416$ 
APPENDIX TABLE A2 - (CONT')

\section{DATE}

$2 / 12 / 91$

$2 / 12 / 91$

$2 / 12 / 91$

$2 / 12 / 91$

$2 / 12 / 91$

$2 / 12 / 91$

$2 / 12 / 91$

$2 / 12 / 91$

$2 / 12 / 91$

$2 / 12 / 91$

$2 / 12 / 91$

$2 / 12 / 91$

$2 / 12 / 91$

$2 / 13 / 91$

$2 / 13 / 91$

$2 / 13 / 91$

$2 / 13 / 91$

$2 / 13 / 91$

$2 / 13 / 91$

$2 / 13 / 91$

$2 / 13 / 91$

$2 / 14 / 91$

$2 / 14 / 91$.

$2 / 14 / 91$

$2 / 14 / 91$

$2 / 14 / 91$

$2 / 14 / 91$

$2 / 14 / 91$

$2 / 14 / 91$

$2 / 14 / 91$

$2 / 14 / 91$

$2 / 14 / 91$

$2 / 14 / 91$

$2 / 14 / 91$

$2 / 14 / 91$

$2 / 15 / 91$

$2 / 15 / 91$

$2 / 15 / 91$

$2 / 15 / 91$

$2 / 15 / 91$

$2 / 15 / 91$

$2 / 15 / 91$
TIME

100

300

500

700

900

1100

1300

1500

1700

1900

2100

2300

MEAN

100

300

500

700

930

1530

2130

MEAN

330

930

1330

1430

1530

1630

1730

1830

1930

2030

2130

2230

2330

MEAN

30

130

230

330

430

530

630
Ts8

(MG/LL

$\begin{array}{ll}25 & 118 \\ 16 & 124 \\ 46 & 424 \\ 26 & 286 \\ 52 & 466 \\ 50 & 460 \\ 53 & 460 \\ 31 & 436 \\ 37 & 436 \\ 26 & 442 \\ 24 & 190 \\ 14 & 158\end{array}$

33

333

144

315

448

448

448

439

445

384

448

436

436

436

436

358

260

341

445

370

322

346

343

383

376

403

394

388

388

388

388 
APPENDIX TABLE A2, (CONT)

DATE

$2 / 15 / 91$

$2 / 15 / 91$

$2 / 15 / 91$

$2 / 15 / 91$

$2 / 15 / 91$

$2 / 15 / 91$

$2 / 15 / 91$

$2 / 15 / 91$

$2 / 15 / 91$

$2 / 16 / 91$

$2 / 16 / 91$

$2 / 16 / 91$

$2 / 16 / 91$

$2 / 16 / 91$

$2 / 16 / 91$

$2 / 16 / 91$

$2 / 16 / 91$

$2 / 16 / 91$

$2 / 17 / 91$

$2 / 17 / 91$

$2 / 17 / 91$

$2 / 17 / 91$

$2 / 17 / 91$

$2 / 17 / 91$

$2 / 17 / 91$

$2 / 17 / 91$

$2 / 17 / 91$

$2 / 18 / 91$

$2 / 1.8 / 91$

$2 / 18 / 91$

$2 / 18 / 91$

$2 / 18 / 91$

$2 / 18 / 91$

$2 / 18 / 91$

$2 / 18 / 91$

$2 / 18 / 91$

$2 / 18 / 91$

$2 / 18 / 91$

$2 / 18 / 91$

$2 / 18 / 91$
TIME

730

830

900

1100

1300

1500

1700

1900

MEAN

100

300

500

700

900

1100

1300

1500

MEAN

1000

1200

1400

1600

1800

2000

2200

2.400

MEAN

200

400

600

800

1000

1200

1400

1600

1800

2000

2200

2.400

MEAN
T88

(MG/L)

$\begin{array}{rr}22 & 388 \\ 20 & 382 \\ 8 & 376 \\ 14 & 376 \\ 11 & 376 \\ 11 & 376 \\ 3 & 376 \\ 2 & 376\end{array}$

18

383

$84 \quad 436$

$92 \quad 436$

$15 \quad 128$

$3 \quad 74$

$7 \quad 76$

$6 \quad 79$

$12 \quad 137$

$7 \quad 130$

$28 \quad 187$

$3 \quad 74$

$9 \quad 90$

$9 \quad 115$

$3 \quad 118$

1.24

124

124

124

112

124

124

127

124

124

130

150

137

137

130

130

158

133 
APPENDIX TABLE A2. (CONT)

\section{DATE}

$2 / 19 / 91$

$2 / 19 / 91$

$2 / 19 / 91$

$2 / 19 / 91$

$2 / 19 / 91$

$2 / 19 / 91$

$2 / 19 / 91$

$2 / 19 / 91$

2/19/91

$2 / 19 / 91$

$2 / 19 / 91$

$2 / 19 / 91$

$2 / 19 / 91$

$2 / 20 / 91$

2/20/91

2/20/91

$2 / 20 / 91$

2/20/91

$2 / 20 / 91$

2/20/91

$2 / 20 / 91$

$2 / 20 / 91$

$2 / 20 / 91$

2/20/91

$2 / 20 / 91$

$2 / 20 / 91$

$2 / 21 / 91$

$2 / 21 / 91$

2/21/91

$2 / 21 / 91$

$2 / 21 / 91$

2/21/91.

$2 / 21 / 91$

2/21/91

2/21/91

2/21/91

$2 / 21 / 91$

2/21/91

$2 / 21 / 91$

2/21/91

$2 / 21 / 91$

$2 / 22 / 91$
TIME

200

400

600

800

930

1130

1330

1530

1730

1930

2130

2330

MEAN

130

330

530

730

930

1130

1330

1530

1730

1.930

2130

2330

MEAN

130

330

530

730

830

900

900

1100

1300

1500

1700

1900

2100

2300

MEAN

100
TS8

(MG/L)

$\begin{array}{rr}11 & 144 \\ 11 & 137 \\ 12 & 140 \\ 13 & 144 \\ 2 & 144 \\ 6 & 142 \\ 5 & 139 \\ 5 & 110 \\ 4 & 121 \\ 4 & 112 \\ 4 & 112 \\ 7 & 149\end{array}$

7

133

173

173

173

173

173

173

171

167

169

167

186

143

170

145

147

142

140

140

130

137

137

124

124

118

1.18

1.15

118

131

115 
APPENDIX TABLE A2. (CONT)

\section{DATE}

$2 / 22 / 91$

$2 / 22 / 91$

$2 / 22 / 91$

$2 / 22 / 91$

$2 / 22 / 91$

$2 / 22 / 91$

$2 / 22 / 91$

$2 / 23 / 91$

$2 / 23 / 91$

$2 / 23 / 91$

$2 / 23 / 91$

$2 / 23 / 91$

$2 / 24 / 91$

$2 / 24 / 91$

$2 / 24 / 91$

$2 / 24 / 91$

$2 / 24 / 91$

$2 / 25 / 91$

$2 / 25 / 91$

$2 / 25 / 91$

$2 / 25 / 91$

$2 / 25 / 91$

$2 / 26 / 91$

$2 / 26 / 91$

$2 / 26 / 91$

$2 / 26 / 91$

$2 / 26 / 91$

$2 / 27 / 91$

$2 / 27 / 91$

$2 / 27 / 91$

$2 / 27 / 91$

$2 / 27 / 91$

$2 / 28 / 91$

$02 / 91$
TIME

300

500

700

900

1500

2100

MEAN

300

900

1500

2100

MEAN

300

900

1500

2100

MEAN

300

900

1500

2100

MEAN

300

900

1500

2100

MEAN

300

900

1500

2100

MEAN

300

AVE

\section{TSS}

$(M G / L)$

FLOW

(CFS)

$\begin{array}{ll}4 & 118 \\ 8 & 165 \\ 5 & 112 \\ 1 & 112 \\ 1 & 112 \\ 1 & 112\end{array}$

3

121

85

95

98

95

93

95

90

90

134

102

150

150

144

144

147

87

85

85

85

85

85

85

79

82

82

85

19

198 
APPENDIX TABLE A2. (CONT)

DATE

$3 / 5 / 91$

$3 / 5 / 91$

$3 / 5 / 91$

$3 / 5 / 91$

$3 / 6 / 91$

$3 / 6 / 91$

$3 / 6 / 91$

$3 / 6 / 91$

$3 / 6 / 91$

$3 / 7 / 91$

$3 / 7 / 91$

$3 / 7 / 91$

$3 / 7 / 91$

$3 / 8 / 91$

$3 / 8 / 91$

$3 / 8 / 91$

$3 / 8 / 91$

$3 / 8 / 91$

$3 / 9 / 91$

$3 / 9 / 91$

$3 / 9 / 91$

$3 / 9 / 91$

$3 / 9 / 91$

$3 / 10 / 91$

$3 / 10 / 91$

$3 / 10 / 91$

$3 / 10 / 91$

$3 / 10 / 91$

$3 / 11 / 91$

$3 / 12 / 91$

$3 / 12 / 91$

$3 / 12 / 91$.

$3 / 12 / 91$
TIME

830

1430

2030

MEAN

230

830

1430

2.030

MEAN

$$
\begin{array}{r}
230 \\
830 \\
2030
\end{array}
$$

MEAN

230

830

1430

2030

MEAN

$$
230
$$

830

1430

2030

MEAN

230

830

1430

2030

MEAN

230

900

1500

2100

MEAN
TS8

(MG/L)

$\begin{array}{ll}52 & 288 \\ 46 & 292 \\ 27 & 292\end{array}$

41

26

40

43

37

36

29

25

32

29

65

109

172

269

153

160

98

97

98

113

59

96

80

61

74

87

69

66

72

69
291

\section{FLOW}

(CF')

294

478

454

460

422

457

337

460

418

460

484

328

321

398

315

328

334

328

326

178

178

169

165

173

154

165

154

154

158 
APPENDIX TABLE A2. (CONT)

\section{DATE}

$3 / 13 / 91$

$3 / 13 / 91$

$3 / 13 / 91$

$3 / 13 / 91$

$3 / 13 / 91$

$3 / 1.4 / 91$

$3 / 14 / 91$

$3 / 14 / 91$

$3 / 14 / 91$

$3 / 14 / 91$

$3 / 15 / 91$

$3 / 15 / 91$

$3 / 15 / 91$

$3 / 15 / 91$

$3 / 15 / 91$

$3 / 16 / 91$

$3 / 16 / 91$

$3 / 16 / 91$

$3 / 16 / 91$

$3 / 16 / 91$

$3 / 17 / 91$

$3 / 17 / 91$

$3 / 17 / 91$

$3 / 17 / 91$

$3 / 17 / 91$

$3 / 18 / 91$

$3 / 1.9 / 91$

$3 / 1.9 / 91$

$3 / 19 / 91$

$3 / 19 / 91$

$3 / 20 / 91$

$3 / 20 / 91$

$3 / 20 / 91$.

$3 / 20 / 91$.

\section{TIME}

300

900

1500

2100

MEAN

300

900

1500

2100

MEAN

$$
\begin{array}{r}
300 \\
900 \\
1500 \\
2100
\end{array}
$$

MEAN

300

900

1500

2100

MEAN

300

900

1500

2100

MEAN

300

900

1500

2100

MEAN

300

900

1500

2100
TSS

$(M G / L)$.

$\begin{array}{ll}86 & 158 \\ 61 & 150 \\ 64 & 218 \\ 68 & 190\end{array}$

69

107

77

74

34

73

19

21

19

21

20

18

23

13

13

16

13

26

42

42

31

61

24

18

17

20

47

20

13

11
179

F'LOW

(CFS)

472

460

460

144

384

1.73

173

1.78

173

174

173

173

1.73

173

173

1.65

292

292

292

260

144

466

442

454

454

292

448

442

448 
APPENDIX TABLE A2. (CONT)

\section{DATE}

$3 / 20 / 91$

$3 / 21 / 91$

$3 / 21 / 91$

$3 / 21 / 91$

$3 / 26 / 91$

$3 / 26 / 91$

$3 / 26 / 91$

$3 / 26 / 91$

$3 / 27 / 91$

$3 / 27 / 91$

$3 / 27 / 91$

$3 / 27 / 91$

$3 / 27 / 91$

$3 / 28 / 91$.

$3 / 28 / 91$

$3 / 28 / 91$

$3 / 28 / 91$

$3 / 28 / 91$

$3 / 29 / 91$

$3 / 29 / 91$

$3 / 29 / 91$

$3 / 29 / 91$.

$3 / 29 / 91$

$3 / 30 / 91$

$3 / 30 / 91$

$3 / 30 / 91$

$3 / 30 / 91$

$3 / 30 / 91$

$3 / 31 / 91$

$3 / 31 / 91$.

$3 / 31 / 91$

$3 / 31 / 91$

$3 / 31 / 91$
TIME

MEAN

300

900

MEAN

900

1500

2100

MEAN

300

900

1500

2100

MEAN

300

900

1500

2100

MEAN

300

900

1500

2100

MEAN

300

900

1500

2100

MEAN

300

900

1500

2100

MEAN
T88

SMG/LL

23

22

74

48

13

12

13

12

16

7

15
7

11

8

1.0

9

6

8

9

2

8

12

8

8

6

5

4

5

6

$\begin{array}{rr}6 & 98 \\ 8 & 101\end{array}$

$9 \quad 98$

9

8

\section{FLOW}

(CFB)

407

460

442

451.

137

130

448

238

448

85

448

448

357

448

442

448

448

446

460

69

79

79

172

98

101

101

101

100

98

1.40

109 
APPENDIX TABLE A2. (CONT)

DATE

$03 / 91$

$4 / 1 / 91$

$4 / 1 / 91$

$4 / 1 / 91$

$4 / 1 / 91$

$4 / 1 / 91$

$4 / 2 / 91$

$4 / 2 / 91$

$4 / 2 / 91$

$4 / 2 / 91$

$4 / 2 / 91$

$4 / 3 / 91$

$4 / 3 / 91$.

$4 / 3 / 91$

$4 / 3 / 91$.

$4 / 3 / 91$

$4 / 4 / 91$

$4 / 4 / 9]$

$4 / 4 / 91$

$4 / 4 / 91$

$4 / 4 / 91$

$4 / 5 / 91$

$4 / 5 / 91$

$4 / 5 / 91$

$4 / 5 / 91$

$4 / 5 / 91$

$4 / 6 / 91$

$4 / 6 / 91$

$4 / 6 / 91$

$4 / 6 / 91$

$4 / 6 / 91$

$4 / 7 / 91$

$4 / 8 / 91$

$4 / 8 / 91$
TIME

AVE

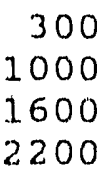

MEAN

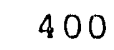

1000

1600

2200

MEAN

400

1000

1600

22,00

MEAN

400

1000

1600

2200

MEAN

400

1000

1600

2200

MEAN

400

1000

1600

2200

MEAN

400

930

1530
TS8

$(M G / L)$

42

13

7

9

9

$7 \quad 112$

1.5

6

10

9

12

22

11

15

15

16

14

16

10

14

12

8

11

9

10

7

8

9

9

8

7

6

5
112

FLOW

(CFS)

285

64

79

112

112

92

112

118

118

115

118

1.15

112

112

115

115

118

115

112

115

118

112

112

106

134

124

124

124

127

124

121

121 
APPENDIX TABLE A2 - (CONT)

\section{DATE}

$4 / 8 / 91$

$4 / 8 / 91$

$4 / 9 / 91$

$4 / 9 / 91$

$4 / 9 / 91$

$4 / 9 / 91$

$4 / 9 / 91$

$4 / 10 / 91$

$4 / 10 / 91$

$4 / 10 / 91$

$4 / 10 / 91$

$4 / 10 / 91$

$4 / 11 / 91$

$4 / 11 / 91$

$4 / 11 / 91$

$4 / 11 / 91$

$4 / 11 / 91$

$4 / 12 / 91$

$4 / 1.2 / 91$

$4 / 12 / 91$

$4 / 12 / 91$

$4 / 12 / 91$

$4 / 13 / 91$

$4 / 13 / 91$.

$4 / 13 / 91$

$4 / 13 / 91$

$4 / 13 / 91$

$4 / 14 / 91$

$4 / 16 / 91$

$4 / 16 / 91$

$4 / 16 / 91$

$4 / 16 / 91$

$4 / 17 / 91$

$4 / 17 / 91$
TIME

2130

MEAN

330

930

1530

2130

MEAN

330

930

1530

2.230

MEAN

330

930

1.530

21.30

MEAN

330

930

1530

2130

MEAN

330

930

1530

2130

MEAN

330

900

1500

2100

MEAN

300

900
T 88

$(M G / L L$

13

8

6

3

4

8

5

6

8

7

12

8

9

4

6

7

6

5

7

6

11

7

6

6
8

8

5

6

3

9

5

9

6

37

9
FLOW

(OFE)

121

121.

1.18

109

101

1.1. 2

11.0

112

1. 1.8

124

118

118

11.8

118

118

118

118

11.2

106

134

315

167

137

124

118

118

124

1. 12

109

112

106

110

106

292 
APPENDIX T'ABLE AZ, (CONT')

\section{DATE}

$4 / 17 / 91$

$4 / 17 / 91$

$4 / 17 / 91$

$4 / 18 / 9.2$

$4 / 18 / 91$

$4 / 18 / 91$

$4 / 18 / 91$

$4 / 18 / 91$

$4 / 19 / 91$

$4 / 19 / 91$.

$4 / 19 / 91$

$4 / 19 / 91$

$4 / 19 / 91$

$4 / 20 / 91$

$4 / 20 / 91$

$4 / 20 / 91$.

$4 / 20 / 91$

$4 / 20 / 91$

$4 / 21 / 91$

$4 / 21,91$.

$1 / 21 / 91$

$4 / 21 / 91$

$4 / 21 / 91$

$4 / 22 / 91$.

$4 / 23 / 91$

$4 / 23 / 91$

$4 / 23 / 91$

$4 / 24 / 91$

$4 / 24 / 91$.

$4 / 24 / 91$

$4 / 24 / 91$

$4 / 24 / 91$

$4 / 25 / 91$

$4 / 25 / 91$
TINㅡㅌㅛ

1500

2100

MEAN

300

900

1500

2100

MEAN

300

900

1500

2100

MEAN

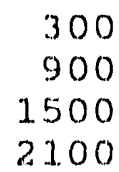

MEAN

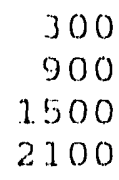

MEAN

$$
\begin{array}{r}
300 \\
1400 \\
2000
\end{array}
$$

MEAN

200

800

1400

2000

MEAN

200

800

\section{8}

$(\mathrm{MG} / \mathrm{L})$

\section{FLOW}

(CF')

13

315

$16 \quad 303$

18

254

21

21

18

16

1.18

448

448

442

19

364

14

16

13

16

15

1.4

12

1.4

5

1.1

270

448

448

448

103

448

436

130

448

365

12

13

8

12

11.

1.1

6

21

13

148

448

454

448

449

448

448

448

448

454

448

448

130

370

9

79

79 
APPENDIX 'TABLE A2, (CON')

\section{DATE}

$4 / 25 / 91$

$4 / 25 / 91$

$4 / 25 / 91$

$4 / 26 / 91$

$4 / 26 / 91$

$4 / 26 / 91$

$4 / 26 / 91$

$4 / 26 / 91$

$4 / 27 / 91$

$4 / 27 / 91$

$4 / 27 / 91$

$1 / 27 / 91$

$4 / 27 / 91$

$4 / 28 / 91$

$4 / 28 / 91$

$4 / 28 / 91$

$4 / 28 / 91$

$4 / 28 / 91$

$4 / 20 / 91$

$4 / 30 / 91$

$1 / 30 / 91$

$4 / 30 / 91$

$04 / 91$

$5 / 1 / 91$

$5 / 1 / 91$

$5 / 1 / 91$

$5 / 1 / 01$

$5 / 1 / 91$

$5 / 2 / 91$

$5 / 2 / 91$

$5 / 2 / 91$

$5 / 2 / 91$

$5 / 2 / 91$
TIME

1400

2000

MLIAN

200

800

1400

2000

MEAN

200

800

1400

2000

MEAN

200

800

1400

2000

MENN

200

800

1400

$M E \Lambda N$

AVE

200

800

1400

2000

MEAN

200

800

1.400

2000

MEAN
T'B8

(MG/L)

3

3

4

8

1

5

6

6

5

4

3

10

5

3
7

11

11

8

15

10

10

10

10

20

9

19

7

1.4

10

5

4

3

5

\section{FLOW}

(CFE).

79

69

$7 \%$

69

69

69

6)

68

$6 \%$

64

64

(i)

64

60

60

240

226

1.46

230

60

62

61.

186

60

60

52

76

62

48

60

62

62

58 


\begin{tabular}{|c|c|c|c|}
\hline DATE & TIME & $\begin{array}{c}T 8 B \\
(M G / L)\end{array}$ & $\begin{array}{l}\text { Y YOW } \\
\text { (CFB) }\end{array}$ \\
\hline $5 / 3 / 91$ & 200 & 6 & 64 \\
\hline $5 / 3 / 91$ & 800 & 5 & 112 \\
\hline $5 / 3 / 91$ & 1400 & 6 & 1.12 \\
\hline $5 / 3 / 91$ & 2000 & 11 & 11.8 \\
\hline $5 / 3 / 91$ & MEAN & 7 & 102 \\
\hline $5 / 1 / 91$ & 200 & 8 & 101 \\
\hline $5 / 4 / 91$ & 800 & 5 & 101 \\
\hline $5 / 4 / 91$ & 1400 & 7 & 101 \\
\hline $5 / 4 / 91$ & 2000 & 2 & 101 \\
\hline $5 / 4 / 91$ & MEAN & 5 & 101 \\
\hline $5 / 5 / 91$ & 200 & 5 & 101 \\
\hline $5 / 5 / 91$ & 800 & 7 & 101 \\
\hline $5 / 5 / 91$ & 1400 & 9 & 101 \\
\hline $5 / 5 / 91$ & 2000 & 8 & 101 \\
\hline $5 / 5 / 91$ & MEAN & 7 & 101 \\
\hline $5 / 6 / 91$ & 200 & 12 & 101 \\
\hline $5 / 7 / 91$ & 800 & 8 & 56 \\
\hline $5 / 7 / 91$ & 1400 & 14 & 52 \\
\hline $5 / 7 / 91$ & 2000 & 7 & 52 \\
\hline $5 / 7 / 91$. & MEAN & 9 & 53 \\
\hline $5 / 8 / 91$ & 200 & 1.6 & 52 \\
\hline $5 / 8 / 91$ & 800 & 4 & 52 \\
\hline $5 / 8 / 91$ & 1400 & 9 & 52 \\
\hline $5 / 8 / 91$ & 2000 & 3 & 52 \\
\hline $5 / 8 / 91$ & MEAN & 8 & 52 \\
\hline $5 / 9 / 91$ & 200 & 7 & 52 \\
\hline $5 / 3 / 91$ & 800 & 5 & 52 \\
\hline $5 / 9 / 92$ & 1400 & 9 & 52 \\
\hline $5 / 9 / 91$ & 2000 & 8 & 52 \\
\hline $5 / 9 / 91$ & MEAN & 7 & 52 \\
\hline $5 / 10 / 91$ & 200 & 4 & 52 \\
\hline $5 / 10 / 91$ & 800 & 8 & 52 \\
\hline $5 / 10 / 91$ & 1400 & 5 & 52 \\
\hline $5 / 10 / 91$ & 2000 & 2 & 52 \\
\hline
\end{tabular}


APPENDIX TABLE A2, (CON'T)

\section{DATE}

$5 / 10 / 91$

$5 / 11 / 91$

$5 / 11 / 91$

$5 / 11 / 91$

$5 / 11 / 91$

$5 / 11 / 91$

$5 / 12 / 91$

$5 / 12 / 91$

$5 / 12 / 91$

$5 / 12 / 91$

$5 / 12 / 91$

$5 / 13 / 91$

$5 / 14 / 91$

$5 / 14 / 91$

$5 / 14 / 91$

$5 / 14 / 91$

$5 / 1.5 / 92$

$5 / 15 / 91$

$5 / 15 / 91$

$5 / 15 / 91$.

$5 / 15 / 91$

$5 / 16 / 91$

$5 / 16 / 91$

$5 / 16 / 91$

$5 / 16 / 91$

$5 / 16 / 91$

$5 / 17 / 91$

$5 / 17 / 91$

$5 / 17 / 91$

$5 / 17 / 91$

$5 / 17 / 91$

$5 / 18 / 91$

$5 / 18 / 91$

$5 / 18 / 91$
IIME

MEAN

200

800

1400

2000

MEAN

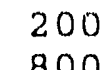

1400

2000

MEAN

200

800

1400

2000

MEAN

200

800

1400

2000

MEAN

200

800

1400

2000

MEAN

200

800

1400

2000

MEAN

200

800

1400
TSS

$(M G / L)$

FLOW

(CFB)

52

52

52

52

52

52

52

52

52

52

52

52

52

54

48

51

48

48

48

48

48

48

48

44

44

46

44

44

48

44

45

44

44

44 


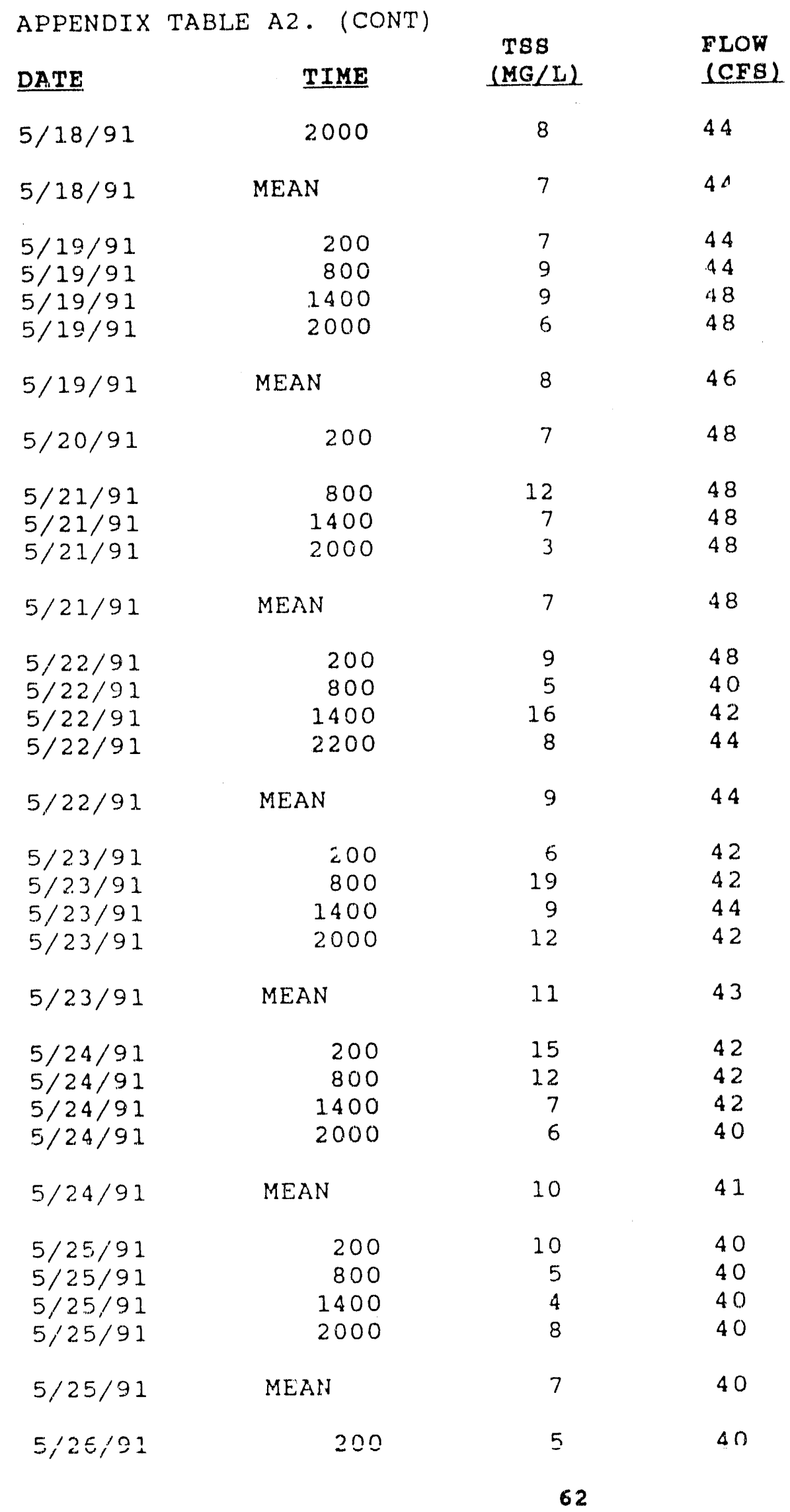


APPENDIX TABLE A2. (CONT)

\section{DATE}

$5 / 26 / 91$

$5 / 26 / 91$

$5 / 26 / 91$

$5 / 26 / 91$

$5 / 27 / 91$

$5 / 28 / 91$

$5 / 28 / 91$

$5 / 28 / 91$

$5 / 29 / 91$

$5 / 29 / 91$

$5 / 29 / 91$

$5 / 29 / 91$

$5 / 29 / 91$

$5 / 30 / 91$

$5 / 30 / 91$

$5 / 30 / 91$

$5 / 30 / 91$

$5 / 30 / 91$

$5 / 31 / 91$

$5 / 31 / 91$

$5 / 31 / 91$

$5 / 31 / 91$

$5 / 31 / 91$

$05 / 91$
TIME

800

1400

2000

MEAN

200

1300

1900

MEAN

100

700

1300

1900

MEAN

100

700

1300

1900

MEAN

100

700

1300

1900

MEAN

AVE
TSS

(MG/L)

6

652

$6 \quad 52$

6

46

44

28

28

28

2.8

28

28

28

28

28

28

28

28

28

13

13

40

40

1126

8

52 
Appendix Table A3. Rainfall in $\mathrm{K}$ Area in Relation to Total suspended solids and Corresponding Flows at Two Locations in the Pen Branch system.

\begin{tabular}{|c|c|c|c|c|c|}
\hline DATE & $\begin{array}{l}\text { PRECIP. } \\
\text { TOTAL }\end{array}$ & $\begin{array}{l}\text { TSS } \\
(\mathrm{mg} / \mathrm{L})\end{array}$ & $\begin{array}{l}\text { TSS } \\
(\mathrm{mg} / \mathrm{L})\end{array}$ & FLOW & $\begin{array}{l}\text { FLOW } \\
\text { (CFS) }\end{array}$ \\
\hline DATE & INCHES & $\mathrm{RD} B$ & RD A 13.2 & $\mathrm{RD} \mathrm{B}$ & RD $A-13.2$ \\
\hline $10 / 1 / 90$ & 0.20 & & & & \\
\hline $10 / 2 / 90$ & 0.00 & & & & \\
\hline $10 / 3 / 90$ & 0.00 & & & & \\
\hline $10 / 4 / 90$ & 0.20 & & & & \\
\hline $10 / 5 / 90$ & 0.03 & & & & \\
\hline $10 / 6 / 90$ & 0.00 & & & & \\
\hline $10 / 7 / 90$ & 0.00 & & & & \\
\hline $10 / 8 / 90$ & 0.00 & & & & \\
\hline $10 / 9 / 90$ & 0.00 & & & & \\
\hline $10 / 20 / 90$ & 4.75 & & & & \\
\hline $10 / 1.1 / 90$ & 2.50 & & & & \\
\hline $10 / 12 / 90$ & 2.50 & 51 & 22 & 52 & 714 \\
\hline $10 / 13 / 90$ & 0.07 & 17 & 60 & 50 & 275 \\
\hline $10 / 14 / 90$ & 0.00 & 23 & & 46 & \\
\hline $10 / 15 / 90$ & 0.00 & 26 & 28 & 51 & 75 \\
\hline $10 / 16 / 90$ & 0.00 & 24 & 23 & 60 & 78 \\
\hline $10 / 17 / 90$ & 0.00 & & & & \\
\hline $10 / 18 / 90$ & 0.15 & & & & \\
\hline $10 / 19 / 90$ & 0.00 & & & & \\
\hline $10 / 20 / 90$ & 0.00 & & & & \\
\hline $10 / 21 / 90$ & 0.00 & & & & \\
\hline $10 / 22 / 90$ & 3.85 & & & & \\
\hline $10 / 23 / 90$ & 0.03 & & & & \\
\hline $10 / 24 / 90$ & 0.20 & & & & \\
\hline $10 / 25 / 90$ & 0.03 & & & & \\
\hline $10 / 26 / 90$ & 0.50 & & & & \\
\hline $10 / 27 / 90$ & 0.00 & & & & \\
\hline $10 / 28 / 90$ & 0.00 & & & & \\
\hline $10 / 29 / 90$ & 0.00 & & & & \\
\hline $10 / 30 / 90$ & 0.00 & & & & \\
\hline $10 / 31 / 90$ & 0.00 & & & & \\
\hline $11 / 1 / 90$ & 0.00 & & & & \\
\hline $12 / 2, / 90$ & 0.00 & & & & \\
\hline $11 / 3 / 90$ & 0.00 & & & & \\
\hline $11 / 4 / 90$ & 0.00 & & & & \\
\hline $11 / 5 / 90$ & 0.00 & & & & \\
\hline $11 / 6 / 90$ & 0.00 & & & & \\
\hline $11 / 7 / 90$ & 0.00 & & & & \\
\hline $11 / 8 / 90$ & 2.25 & & & & \\
\hline $11 / 9 / 90$ & 0.00 & & & & \\
\hline $11 / 10 / 90$ & 0.00 & & & & \\
\hline $1.1 / 11 / 90$ & 0.00 & & & & \\
\hline $11 / 12 / 90$ & 0.00 & & & & \\
\hline $11 / 13 / 90$ & 0.00 & & & & \\
\hline $11 / 14 / 90$ & 0.00 & & & & \\
\hline
\end{tabular}




\begin{tabular}{|c|c|c|c|c|c|}
\hline DATE & $\begin{array}{l}\text { PRECIP. } \\
\text { TOTAL }\end{array}$ & $\begin{array}{l}\text { TSS } \\
(\mathrm{mg} / \mathrm{L})\end{array}$ & $\begin{array}{l}\text { TSS } \\
(\mathrm{mg} / \mathrm{L})\end{array}$ & $\begin{array}{l}\text { FLOW } \\
\text { (CFS) }\end{array}$ & $\begin{array}{l}\text { FLOW } \\
\text { (CFS) }\end{array}$ \\
\hline DATE & INCHES & $\mathrm{RD} \mathrm{B}$ & $\mathrm{RD} A 13.2$ & $\mathrm{RD} B$ & RD $A-13.2$ \\
\hline $11 / 15 / 90$ & 0.00 & & & & \\
\hline $11 / 16 / 90$ & 0.00 & & & & \\
\hline $11 / 1.7 / 90$ & 0.00 & & & & \\
\hline $11 / 18 / 90$ & 0.00 & & & & \\
\hline $11 / 19 / 90$ & 0.00 & & & & \\
\hline $11 / 20 / 90$ & 0.00 & & & & \\
\hline $11 / 21 / 90$ & 0.00 & & & & \\
\hline $11 / 22 / 90$ & 0.00 & & & & \\
\hline $11 / 23 / 90$ & 0.00 & & & & \\
\hline $11 / 24 / 90$ & 0.00 & & & & \\
\hline $11 / 25 / 90$ & 0.00 & & & & \\
\hline $11 / 26 / 90$ & 0.00 & & & & \\
\hline $11 / 27 / 90$ & 0.05 & & & & \\
\hline $11 / 28 / 90$ & 0.14 & 4 & 57 & 48 & 78 \\
\hline $11 / 29 / 90$ & 0.00 & 4 & 25 & 48 & 84 \\
\hline $11 / 30 / 90$ & 0.00 & 4 & 3 & 64 & 74 \\
\hline $12 / 01 / 90$ & 0.00 & 4 & 4 & 60 & 78 \\
\hline $12 / 02 / 90$ & 0.00 & 7 & 5 & 60 & 67 \\
\hline $12 / 03 / 90$ & 0.00 & 8 & 7 & 60 & 69 \\
\hline $12 / 04 / 90$ & 0.00 & 22 & 14 & 60 & 69 \\
\hline $12 / 05 / 90$ & 0.00 & 22 & 7 & 62 & 68 \\
\hline $12 / 06 / 90$ & 0.00 & & 11 & & 67 \\
\hline $12 / 07 / 90$ & 0.55 & & 21 & & 78 \\
\hline $12 / 08 / 90$ & 0.00 & & & & \\
\hline $12 / 09 / 90$ & 0.00 & & & & \\
\hline $12 / 10 / 90$ & 0.00 & 5 & 10 & 74 & 84 \\
\hline $12 / 11 / 90$ & 0.00 & 8 & 16 & 69 & 78 \\
\hline $12 / 12 / 90$ & 0.00 & 7 & 12 & 69 & 74 \\
\hline $12 / 13 / 90$ & 0.00 & 7 & 14 & 65 & 74 \\
\hline $12 / 14 / 90$ & 0.00 & 8 & 15 & 70 & 77 \\
\hline $12 / 15 / 90$ & 0.05 & 8 & 14 & 68 & 77 \\
\hline $12 / 16 / 90$ & 0.00 & 8 & 12 & 66 & 76 \\
\hline $12 / 17 / 90$ & 0.00 & & & & \\
\hline $12 / 18 / 90$ & 0.10 & 14 & 14 & 79 & 90 \\
\hline $12 / 19 / 90$ & 0.20 & 11. & 21. & 78 & 92 \\
\hline $12 / 20 / 90$ & 0.30 & 14 & 32 & 77 & 93 \\
\hline $12 / 21 / 90$ & 0.05 & 13 & 28 & 69 & 91 \\
\hline $12 / 22 / 90$ & 0.00 & 15 & 27 & 64 & 80 \\
\hline $12 / 23 / 90$ & 0.05 & 13 & 23 & 64 & 76 \\
\hline $12 / 24 / 90$ & 0.00 & & & & \\
\hline $12 / 25 / 90$ & 0.00 & & & & \\
\hline $12 / 26 / 90$ & 0.00 & & & & \\
\hline $12 / 27 / 90$ & 0.01 & & & & \\
\hline $12 / 28 / 90$ & & & & & \\
\hline $12 / 29 / 90$ & 0.05 & & & & \\
\hline
\end{tabular}




$\begin{array}{llllll}\text { APPENDIX TABLE } & \text { A-3 } & \text { (CONT.) } & & & \\ \text { DATE } & \text { PRECIP. } & \text { TSS } & \text { TSS } & \text { FLOW } & \text { FLOW } \\ & \text { TOTAL } & (\mathrm{mg} / \mathrm{L}) & (\mathrm{mg} / \mathrm{L}) & \text { (CFS) } & \text { (CFS) } \\ \text { DATE } & \text { INCHES } & \text { RD B } & \text { RD A13.2 } & \text { RD B } & \text { RD A-13.2 }\end{array}$

$$
\begin{aligned}
& 12 / 30 / 90 \\
& 12 / 31 / 90 \\
& 01 / 01 / 91 \\
& 01 / 02 / 91 \\
& 01 / 03 / 91 \\
& 01 / 04 / 91 \\
& 01 / 05 / 91 \\
& 01 / 06 / 91 \\
& 01 / 07 / 91 \\
& 01 / 08 / 91 \\
& 01 / 09 / 91 \\
& 01 / 10 / 91 \\
& 01 / 11 / 91 \\
& 01 / 12 / 91 \\
& 01 / 13 / 91 \\
& 01 / 14 / 91 \\
& 01 / 15 / 91 \\
& 01 / 16 / 91 \\
& 01 / 17 / 91 \\
& 01 / 18 / 91 \\
& 01 / 19 / 91 \\
& 01 / 20 / 91 \\
& 01 / 21 / 91 \\
& 1 / 22 / 91 \\
& 1 / 23 / 91 \\
& 1 / 24 / 91 \\
& 1 / 25191 \\
& 1 / 26 / 91 \\
& 1 / 27191 \\
& 1 / 28 / 91 \\
& 1 / 29 / 91 \\
& 1 / 30 / 91 \\
& 1 / 31 / 91 \\
& 2 / 1 / 91 \\
& 2 / 2 / 91 \\
& 2 / 3 / 91 \\
& 2 / 4 / 91 \\
& 2 / 5 / 91 \\
& 2 / 6 / 91 \\
& 2 / 7 / 91 \\
& 2 / 8 / 91 \\
& 2 / 9 / 91 \\
& 2110 / 91 \\
& 2 / 11191 \\
& 2 / 12 / 91
\end{aligned}
$$

0.05

0.00

0.40

0.02

0.12

0.01

0.00

0.00

0.17

0.03

0.00

0.1 .5

1.00

0.00

0.00

0.00

0.40

0.04

0.05

0.05

1. 25

0.00

0.00

0.00

0.15

I. 00

0.10

0.00

0.00

0.10

0.80

1.25

0.00

0.00

0.00

0.00

0.00

0.00

0.80

0.41

0.00

0.00

0.00

$\begin{array}{rrr}9 & 74 & 93 \\ 9 & 75 & 88 \\ 13 & 78 & 147 \\ 11 & 78 & 140 \\ 7 & 78 & 106 \\ 6 & 76 & 100 \\ & 76 & \\ 12 & 74 & 104 \\ 34 & 73 & 355 \\ 15 & 77 & 199 \\ 14 & 74 & 123 \\ 13 & 75 & 108 \\ 11 & 74 & 104 \\ 9 & 74 & 101 \\ 8 & 74 & 97 \\ 12 & 91 & 108 \\ 18 & 148 & 258 \\ 13 & 148 & 231 \\ 11 & 164 & 203 \\ 45 & 284 & 273 \\ 56 & 416 & 527 \\ 41 & 333 & 377\end{array}$


APPENDIX TABLE A-3 (CONT.)

\begin{tabular}{|c|c|c|c|c|c|}
\hline DATE & $\begin{array}{l}\text { PRECIP. } \\
\text { TOTAL }\end{array}$ & TSS & $\begin{array}{l}\text { TSS } \\
(\mathrm{mg} / \mathrm{L})\end{array}$ & $\begin{array}{l}\text { FLOW } \\
\text { (CFS) }\end{array}$ & $\begin{array}{l}\text { FLOW } \\
\text { (CFS) }\end{array}$ \\
\hline DATE & INCHES & $R D \quad B$ & RD $A 13.2$ & $\mathrm{RD} \mathrm{B}$ & RD $A-13.2$ \\
\hline $2 / 13 / 91$ & 0.02 & 25 & 30 & 384 & 347 \\
\hline $2 / 14 / 91$ & 0.00 & 27 & 21 & 383 & 462 \\
\hline $2 / 15 / 91$ & 0.00 & 18 & 21 & 383 & 412 \\
\hline $2 / 16 / 91$ & 0.00 & 28 & & 187 & \\
\hline $2 / 17 / 91$ & 0.00 & 7 & & 112 & \\
\hline $2 / 18 / 91$ & 0.00 & 8 & & 133 & \\
\hline $2 / 19 / 91$ & 0.00 & 7 & 10 & 133 & 138 \\
\hline $2 / 20 / 91$ & 0.00 & 7 & 17 & 170 & 179 \\
\hline $2 / 21 / 91$. & 0.00 & 4 & 7 & 131 & 146 \\
\hline $2 / 22 / 91$ & 0.60 & 3 & 8 & 121 & 127 \\
\hline $2 / 23 / 91$ & 0.60 & 4 & 14 & 93 & 126 \\
\hline $2 / 24 / 91$ & 0.03 & 4 & 11 & 102 & 107 \\
\hline $2 / 25 / 91$ & 0.00 & 3 & 17 & 147 & 160 \\
\hline $2 / 26 / 91$ & 0.00 & 1 & 13 & 85 & 101 \\
\hline $2 / 27 / 91$ & 0.00 & 4 & 7 & 82 & 92 \\
\hline $2 / 28 / 91$ & 0.00 & 2 & 4 & 85 & 83 \\
\hline $3 / 1 / 91$ & 1.25 & & & & \\
\hline $3 / 2 / 91$ & 0.70 & & & & \\
\hline $3 / 3 / 91$ & 1.65 & & & & \\
\hline $3 / 4 / 91$ & 0.00 & & & & \\
\hline $3 / 5 / 91$ & 0.00 & 41 & 29 & 291 & 353 \\
\hline $3 / 6 / 91$ & 0.00 & 36 & 24 & 422 & 445 \\
\hline $3 / 7 / 91$ & 0.00 & 29 & 23 & 418 & 381 \\
\hline $3 / 8 / 91$ & 0.07 & 153 & 19 & 398 & 356 \\
\hline $3 / 9 / 91$ & 0.00 & 1.13 & 12 & 326 & 246 \\
\hline $3 / 10 / 91$ & 0.01 & 74 & 14 & 173 & 266 \\
\hline $3 / 11 / 91$ & 0.00 & 87 & 12 & 154 & 176 \\
\hline $3 / 12 / 91$ & 1.70 & 69 & 15 & 158 & 210 \\
\hline $3 / 13 / 91$ & 0.52 & 69 & 18 & 179 & 215 \\
\hline $3 / 14 / 91$ & 0.03 & 73 & 11. & 384 & 329 \\
\hline $3 / 15 / 91$ & 0.00 & 20 & 8 & 174 & 185 \\
\hline $3 / 16 / 91$ & 0.00 & .26 & 4 & 173 & 218 \\
\hline $3 / 17 / 91$ & 0.04 & 31 & 10 & 260 & 225 \\
\hline $3 / 18 / 91$ & 0.15 & 61 & 5 & 144 & 417 \\
\hline $3 / 19 / 91$ & 0.00 & 20 & 21 & 454 & 453 \\
\hline $3 / 20 / 91$ & 0.00 & 23 & 16 & 407 & 352 \\
\hline $3 / 21 / 91$ & 0.00 & 48 & 17 & 451 & 372 \\
\hline $3 / 22 / 91$ & 0.00 & & 13 & & 398 \\
\hline $3 / 23 / 91$ & 0.00 & & 15 & & 188 \\
\hline $3 / 24 / 91$ & 0.00 & & 12 & & 72 \\
\hline $3 / 25 / 91$ & 0.00 & & 19 & & 67 \\
\hline $3 / 26 / 91$ & 0.00 & 12 & 27 & 238 & 245 \\
\hline $3 / 27 / 91$ & 0.00 & 11 & 26 & 357 & 355 \\
\hline $3 / 28 / 91$ & 0.00 & 8 & 17 & 446 & 373 \\
\hline $3 / 29 / 91$ & 2.25 & 8 & 26 & 172 & 272 \\
\hline
\end{tabular}


APPENDIX TABLE A-3 (CONT.)

\section{DATE}

DATE

$3 / 30 / 91$

$3 / 31 / 91$

$4 / 1 / 91$

$4 / 2 / 91$

$4 / 3 / 91$

$4 / 4 / 91$

$4 / 5 / 91$

$4 / 6 / 91$

$4 / 7 / 91$.

$4 / 8 / 91$

$4 / 9 / 91$

$4 / 10 / 91$

$4 / 11 ; 91$

$4 / 12 / 91$

$4 / 13 / 91$

$4 / 14 / 91$

$4 / 15 / 91$

$4 / 16 / 91$

$4 / 17 / 91$

$4 / 18 / 91$

$4 / 19 / 91$

$4 / 20 / 91$

$4 / 21 / 91$

$4 / 22 / 91$

$4 / 23 / 91$

$4 / 24 / 91$

$4 / 25 / 91$

$4 / 26 / 91$

$4 / 27 / 91$

$4 / 28 / 91$

$4 / 29 / 91$

$4 / 30 / 91$

$5 / 1 / 91$

$5 / 2 / 91$

$5 / 3 / 91$

$5 / 4 / 91$

$5 / 5 / 91$

$5 / 6 / 91$

$5 / 7 / 91$

$5 / 8 / 91$

$5 / 9 / 91$.

$5 / 10 / 91$

$5 / 11 / 91$

$5 / 12 / 91$

$5 / 13 / 91$
PRECIP. TSS

TOTAL

INCHES

$(\mathrm{mg} / \mathrm{L})$

TSS

$(\mathrm{mg} / \mathrm{L})$

FLOW

(CFS)

$\mathrm{RD} \mathrm{B} \quad \mathrm{RDA} \mathrm{A} 3.2 \quad \mathrm{RD} B$

0.03

0.00

0.00

0.00

0.00

0.00

1.27

0.00

0.02

0.30

0.07

0.00

0.02

0.00

0.00

0.00

0.15

0.00

0.00

0.00

0.48

0.00

0.00

0.03

0.00

0.00

0.00

1.00

2.00

0.14

0.91

0.92

0.15

0.00

0.00

0.30

1.00

0.05

0.00

0.00

0.20

0.00

0.00

0.19

0.40

\section{5}

8

9

9

15

14

10

8

8
7

8

5

8

6

7

6

3

6

18

19

15

11.

11

11

13

9

4

6

5

8

15

10

14

5

7

5

7

12

9

8

7

5

3

4
16

16

15

16

22.

16

23

20

10

1.1

12

10

12

21.

11

11

15

12

9

8

12

13

13

8

8

24

13

13

15

11

11

8

6

13

$$
7
$$

7

11

8

8
10

10
7
100

109

92

115

115

115

112

127

124

121

110

118

118

167

124

112

110

254

364

403

365

449

448

448

370

77

68

64

146

230

61

62

58

102

101

101.

101

53

52

52

52

52

52

52
FLOW

(CF'S)

RD $A-13.2$

226

149

123

130

129

1.26

183

229

147

150

141

127

180

139

124

124

191

341

357

318

379

357

370

77

64

100

219

208

207

101

82

122

120

166

119

73

67

69

62

58

63 


\begin{tabular}{|c|c|c|c|c|c|}
\hline DATE & $\begin{array}{l}\text { PRECIP. } \\
\text { TOTAL }\end{array}$ & $\begin{array}{l}\text { TSS } \\
(\mathrm{mg} / \mathrm{L})\end{array}$ & $\begin{array}{l}\text { TSS } \\
(\mathrm{mg} / \mathrm{L})\end{array}$ & $\begin{array}{l}\text { FLOW } \\
\text { (CFS) }\end{array}$ & $\begin{array}{l}\text { FLOW } \\
\text { (CFS) }\end{array}$ \\
\hline DATE & INCHES & $\mathrm{RD} \mathrm{B}$ & RD $\mathrm{A} 13.2$ & $\mathrm{RD} \quad \mathrm{B}$ & RD $A-13.2$ \\
\hline $5 / 14 / 91$ & 0.01 & 16 & 17 & 51 & 83 \\
\hline $5 / 15 / 91$ & 0.00 & 16 & 16 & 48 & 61 \\
\hline $5 / 16 / 91$ & 0.00 & 9 & 19 & 46 & 50 \\
\hline $5 / 17 / 91$ & 0.00 & 6 & 19 & 45 & 47 \\
\hline $5 / 18 / 91$ & 0.00 & 7 & 21 & 44 & 45 \\
\hline $5 / 19 / 91$ & 0.00 & 8 & & 46 & \\
\hline $5 / 20 / 91$ & 0.02 & 7 & & 48 & \\
\hline $5 / 21 / 91$ & 0.05 & 7 & 33 & 48 & 53 \\
\hline $5 / 22 / 91$ & 0.00 & 9 & 21 & 44 & 47 \\
\hline $5 / 23 / 91$ & 0.00 & 11 & 23 & 43 & 45 \\
\hline $5 / 24 / 91$ & 0.00 & 10 & 13 & 41 & 47 \\
\hline $5 / 25 / 91$ & 0.00 & 7 & 39 & 40 & 39 \\
\hline $5 / 26 / 91$ & 0.00 & 6 & 26 & 46 & 38 \\
\hline $5 / 27 / 91$ & 0.09 & 8 & & 44 & \\
\hline $5 / 28 / 91$ & 0.00 & 5 & 22 & 28 & 42 \\
\hline $5 / 29 / 91$ & & 4 & 21 & 28 & 34 \\
\hline $5 / 30 / 91$ & & 6 & 16 & 28 & \\
\hline $5 / 31 / 91$ & 0.13 & 11 & & 26 & 46 \\
\hline
\end{tabular}


Appendix Table A4. USG8 Flow Measurements (Provisional Data). Daily Averages at Threo Gaging stations; Indian Grave Branch above Road $B$ (IGB), K-Reactor Cooling Water Discharge Canal $(K-011)$, and Pen Branch at Road $A-13.2$.

\begin{tabular}{|c|c|c|c|}
\hline $\begin{array}{l}\text { DATE } \\
1990\end{array}$ & $\begin{array}{l}\text { IGB } \\
\text { CFB) }\end{array}$ & $\begin{array}{l}X-011 \\
(C F 8)\end{array}$ & $\begin{array}{l}\mathrm{RD} A-13.2 \\
(\mathrm{CFB})\end{array}$ \\
\hline$\frac{1990}{10 / 1}$ & $\frac{0.01}{0.31}$ & $\frac{10101}{38}$ & $\frac{10 \mathrm{LL}}{39}$ \\
\hline $10 / 2$ & 0.30 & 35 & 30 \\
\hline $10 / 3$ & 0.29 & 43 & 34 \\
\hline $10 / 4$ & 0.30 & 48 & 44 \\
\hline $10 / 5$ & 0.32 & 47 & 44 \\
\hline $10 / 6$ & 0.30 & 49 & 45 \\
\hline $10 / 7$ & 0.30 & 48 & 45 \\
\hline $10 / 8$ & 0.31 & 47 & 45 \\
\hline $10 / 9$ & 0.32 & 48 & 44 \\
\hline $10 / 10$ & & 50 & 102 \\
\hline $10 / 11$ & & 51 & 263 \\
\hline $10 / 12$ & & 52 & 357 \\
\hline $10 / 13$ & 1.20 & 49 & 258 \\
\hline $10 / 14$ & 0.39 & 46 & 100 \\
\hline $10 / 15$ & 0.25 & 50 & 79 \\
\hline $10 / 16$ & 0.22 & 62 & 86 \\
\hline $10 / 17$ & 0.26 & 55 & 76 \\
\hline $10 / 18$ & 0.29 & 58 & 82 \\
\hline $10 / 1.9$ & 0.27 & 52 & 74 \\
\hline $10 / 20$ & 0.26 & 51 & 69 \\
\hline $10 / 21$ & 0.28 & 50 & 66 \\
\hline $10 / 22$ & & 48 & 101 \\
\hline $10 / 23$ & & 47 & 562 \\
\hline $10 / 24$ & 2.60 & 50 & 164 \\
\hline $10 / 25$ & 1.90 & 50 & 104 \\
\hline $10 / 26$ & 1.80 & 48 & 84 \\
\hline $10 / 27$ & 1.70 & 49 & 77 \\
\hline $10 / 28$ & 1.50 & 49 & 70 \\
\hline $10 / 29$ & 1.40 & 48 & 67 \\
\hline $10 / 30$ & 1.40 & 50 & 66 \\
\hline $10 / 31$ & 1.30 & 49 & 64 \\
\hline $11 / 1$ & 1.30 & 51 & 63 \\
\hline $1.1 / 2$ & 1.40 & 57 & 66 \\
\hline $11 / 3$ & 1.40 & 59 & 72 \\
\hline $11 / 4$ & 1.50 & 40 & 57 \\
\hline $11 / 5$ & 1.50 & 37 & 49 \\
\hline $11 / 6$ & 1.70 & 37 & 47 \\
\hline $11 / 7$ & 1.70 & 40 & 48 \\
\hline $11 / 8$ & 1.80 & 47 & 54 \\
\hline $11 / 9$ & & 47 & 67 \\
\hline $11 / 10$ & & 46 & 110 \\
\hline $11 / 11$ & 2.40 & 45 & 76 \\
\hline
\end{tabular}




\begin{tabular}{|c|c|c|c|}
\hline $\begin{array}{l}\text { DATE } \\
1990 \\
\end{array}$ & $\begin{array}{l}\text { IGB } \\
\text { CFB) }\end{array}$ & $\begin{array}{l}K-011 \\
(C F B)\end{array}$ & $\begin{array}{c}\text { RD } A-1.3 .2 \\
(C F B)\end{array}$ \\
\hline $11 / 12$ & 2.50 & 46 & 66 \\
\hline $11 / 13$ & 2.80 & 47 & 64 \\
\hline $11 / 14$ & 3.40 & 47 & 63 \\
\hline $11 / 15$ & 4.00 & 48 & 63 \\
\hline $11 / 16$ & 3.20 & 47 & 61 \\
\hline $11 / 17$ & 1.70 & 48 & 61 \\
\hline $11 / 18$ & 2.70 & 53 & 64 \\
\hline $11 / 19$ & & 54 & 68 \\
\hline $11 / 20$ & & 51 & 64 \\
\hline $1.1 / 21$ & & 51 & 65 \\
\hline $1.1 / 22$ & & 51 & 65 \\
\hline $11 / 23$ & & 51 & 65 \\
\hline $11 / 24$ & & 51 & 65 \\
\hline $11 / 25$ & & 52 & 65 \\
\hline $1.1 / 26$ & & 53 & 63 \\
\hline $11 / 27$ & & 60 & 66 \\
\hline $11 / 28$ & & 69 & 78 \\
\hline $1.1 / 29$ & & 69 & 80 \\
\hline $11 / 30$ & & 66 & 76 \\
\hline $12 / 1$ & & 65 & 75 \\
\hline $12 / 2$ & & 59 & 69 \\
\hline $12 / 3$ & & 60 & 69 \\
\hline $12 / 4$ & & 60 & 72 \\
\hline $12 / 5$ & & 60 & 70 \\
\hline $12 / 6$ & & 62 & 69 \\
\hline $12 / 7$ & & 75 & 83 \\
\hline $12 / 8$ & & 75 & 99 \\
\hline $12 / 9$ & & 72 & 89 \\
\hline $12 / 10$ & & 72 & 83 \\
\hline $12 / 11$ & & 69 & 80 \\
\hline $21 / 12$ & & 69 & 77 \\
\hline $.22 / 13$ & & 65 & 75 \\
\hline $12 / 14$ & & 69 & 78 \\
\hline $12 / 15$ & & 69 & 78 \\
\hline $12 / 16$ & & 65 & 76 \\
\hline $12 / 17$ & & 73 & 81. \\
\hline $12 / 18$ & & 79 & 88 \\
\hline $12 / 19$ & & 77 & 90 \\
\hline $12 / 20$ & & 74 & 91 \\
\hline $12 / 21$ & & 70 & 91. \\
\hline $12 / 22$ & & 64 & 80 \\
\hline $12 / 23$ & & 64 & 77 \\
\hline $1.2 / 24$ & & 58 & 70 \\
\hline $12 / 25$ & & 53 & 63 \\
\hline
\end{tabular}


Appendix Table A4 (Cont.)

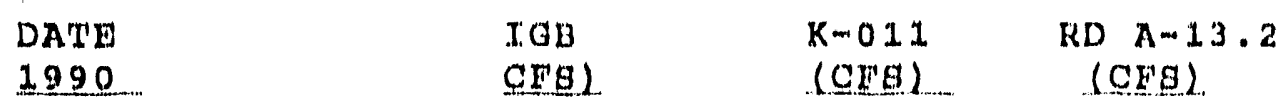

$12 / 26$

$12 / 27$

$12 / 28$

$12 / 29$

$12 / 30$

$12 / 31$

$\begin{array}{ll}44 & 54 \\ 31 & 41 \\ 29 & 38 \\ 28 & 37 \\ 28 & 37 \\ 28 & 38\end{array}$

1991

$1 / .1$

$1 / 2$

$1 / 3$

$1 / 4$

$1 / 5$

$1 / 6$

1.7

$1 / 8$

$1 / 9$

$1 / 10$

$1 / 11$

$1 / 12$

$1 / 13$

$1 / 14$

$1 / 15$

$1 / 16$

$1 / 17$

$1 . / 18$

$1 / 19$

$1 / 20$

1.21 .

$1 / 22$

$1 / 23$

$1 / 24$

1.25

$1 . / 26$

$1 / 27$

$1 / 28$

$1 / 29$

$1 / 30$

$1 / 31$

$2 / 1$

0.43

0.37

0.35

$2 / 3$

$2 / 4$

0.33

$2 / 5$

0.33

$\begin{array}{rr}28 & \\ 31 & 39 \\ 31 & 48 \\ 31 & 45 \\ 44 & 44 \\ 51 & 51 \\ 51 & 60 \\ 51 & 60 \\ 51 & 64 \\ 51 & 61 \\ 51 & 60 \\ 51 & 87 \\ 53 & 96 \\ 51 & 71 \\ 45 & 64 \\ 33 & 60 \\ 54 & 55 \\ 54 & 69 \\ 81 & 67 \\ 75 & 110 \\ 73 & 150 \\ 74 & 104 \\ 76 & 93 \\ 77 & 89 \\ 76 & 131 \\ 77 & 149 \\ 75 & 106 \\ 74 & 98 \\ 73 & 100 \\ 73 & 99 \\ 76 & 292 \\ 76 & 216 \\ 74 & 127 \\ 74 & 107 \\ 73 & 102 \\ 73 & 98 \\ 89 & 95 \\ & 103\end{array}$




\begin{tabular}{|c|c|c|c|}
\hline $\begin{array}{l}\text { DATE } \\
1990 \\
\end{array}$ & $\begin{array}{l}\mathrm{T} Q \mathrm{~B} \\
\mathrm{CFB} \text {. }\end{array}$ & $\begin{array}{l}K-011 \\
\left(C E^{\prime} B\right)\end{array}$ & $\begin{array}{c}\text { RD } A-13.2 \\
(\text { CF } 9)\end{array}$ \\
\hline $2 / 7$ & & 148 & 258 \\
\hline $2 / 8$ & & 148 & 234 \\
\hline $2 / 9$ & & 161 & 206 \\
\hline $2 / 10$ & & 283 & 305 \\
\hline $2 / 11$ & 0.43 & 41.8 & 431 \\
\hline $2 / 12$ & & 327 & 370 \\
\hline $2 / 13$ & 0.43 & 411. & 390 \\
\hline $2 / 14$ & 0.29 & 41.2 & 450 \\
\hline $2 / 15$ & 0.25 & 388 & 388 \\
\hline $2 / 16$ & 0.24 & 156 & 221 \\
\hline $2 / 17$ & 0.24 & 99 & 105 \\
\hline $2 / 18$ & 0.27 & 136 & 146 \\
\hline $2 / 19$ & & 132 & 150 \\
\hline $2 / 20$ & & 171 & 1.81 \\
\hline $2 / 21$ & 0.25 & 133 & 151 \\
\hline $2 / 22$ & 0.25 & 115 & 125 \\
\hline $2 / 23$ & 0.40 & 97 & 124 \\
\hline $2 / 24$ & 0.27 & 97 & 109 \\
\hline $2 / 25$ & 0.24 & 141 & 161 \\
\hline $2 / 26$ & 0.26 & 86 & 99 \\
\hline $2 / 27$ & 0.28 & 82 & 90 \\
\hline $2 / 28$ & 0.25 & 109 & 110 \\
\hline $3 / 1$ & 0.29 & 143 & 154 \\
\hline $3 / 2$ & 2.00 & 112 & 2. 1.4 \\
\hline $3 / 3$ & & 124 & 337 \\
\hline $3 / 4$ & 1.40 & 275 & 407 \\
\hline $3 / 5$ & & 292 & 340 \\
\hline $3 / 6$ & & 424 & 411. \\
\hline $3 / 7$ & & 430 & 436 \\
\hline $3 / 8$ & & 384 & 420 \\
\hline $3 / 9$ & & 326 & 331 \\
\hline $3 / 10$ & & 180 & 221 \\
\hline $3 / 11$ & & 211 & 220 \\
\hline $3 / 12$ & & 168 & 182 \\
\hline $3 / 13$ & & 169 & 196 \\
\hline $3 / 14$ & & 372 & 379 \\
\hline $3 / 15$ & & 172 & 186 \\
\hline $3 / 16$ & & 1.73 & 182 \\
\hline $3 / 17$ & & 268 & 251 \\
\hline $3 / 18$ & & 169 & 203 \\
\hline $3 / 19$ & & 410 & 356 \\
\hline $3 / 20$ & & 41.8 & 423 \\
\hline $3 / 21$ & & 438 & 431 \\
\hline $3 / 22$ & 0.38 & 451 & 452 \\
\hline $3 / 23$ & 0.43 & 168 & 240 \\
\hline
\end{tabular}


Appendix 'lable A4 (Cont.)

$\begin{array}{llll}\text { DATE } & \text { IGB } & K-011 & R D A-13.2 \\ 1990 & \text { CFE) } & \text { (CFE) } & (C F B)\end{array}$

$3 / 24$

$3 / 25$

$3 / 26$

$3 / 27$

$3 / 28$

$3 / 29$

3,30

$3 / 31$

$4 / 1$

$4 / 2$

$4 / 3$

$4 / 4$

$4 / 5$

$4 / 6$

$4 / 7$

$4 / 8$

$4 / 9$

$4 / 10$

$4 / 11$

$4 / 12$

$4 / 13$

$4 / 14$

$4 / 15$

$4 / 16$

$4 / 17$

$4 / 18$

$4 / 19$

$4 / 20$

$4 / 21$.

$4 / 22$

$4 / 23$

$4 / 24$

$4 / 25$

$4 / 26$

$4 / 27$

$4 / 28$

$4 / 29$

$4 / 30$

$5 / 1$.

$5 / 2$

$5 / 3$

$5 / 4$

$5 / 5$

$5 / 6$

$5 / 7$
0.43

0.30

0.38

0.38

0.66

0.58

0.53

0.50

0.50

0.76

0.59

0.66

0.74

0.63

0.58

0.56

0.56

0.56

0.53

0.55

0.52

0.52

0.71

0.59

0.55

0.53

0.54

0.48

0.41

0.50

0.85

0.70

0.82

0.70

1. 10
58

56

205

419

448

168

100

1.09

96

114

114

115

113

1.28

123

120

112

117

118

1.80

141

112

112

107

228

360

411

350

448

450

445

386

78

68

63

148

220

6.1

71

62

102

101

101

101

80
76

71.

144

418

433

285

236

133

1.13

120

120

11.8

151

194

130

134

129

125

163

149

115

1.28

114

216

337

390

355

435

436

429

402

85

69

96

207

255

116

88

74

99

101.

128

1.67

101 
Appendix l'able A4 (Cont.)

\begin{tabular}{|c|c|c|c|}
\hline $\begin{array}{l}\text { DATE } \\
1990 \\
\end{array}$ & $\begin{array}{l}T \in B \\
\text { CFBL }\end{array}$ & $\begin{array}{r}K-011 \\
(058)\end{array}$ & $\begin{array}{l}\text { RD A- } \\
\text { LCF }\end{array}$ \\
\hline $5 / 8$ & & 52 & 64 \\
\hline $5 / 9$ & 1.30 & 51. & 61 \\
\hline $5 / 10$ & 1.10 & 51 & 61 \\
\hline $5 / 11$ & 0.58 & 51 & 56 \\
\hline $5 / 12$ & 0.61 & 51 & 55 \\
\hline $5 / 13$ & 0.80 & 52 & 76 \\
\hline $5 / 14$ & 0.65 & 51. & 75 \\
\hline $5 / 15$ & 0.57 & 47 & 55 \\
\hline $5 / 16$ & 0.56 & 45 & 48 \\
\hline $5 / 17$ & 0.55 & 46 & 47 \\
\hline $5 / 18$ & 0.51 & 45 & 45 \\
\hline $5 / 19$ & 0.52 & 45 & 47 \\
\hline $5 / 20$ & 0.57 & 45 & 48 \\
\hline $5 / 21$ & 0.56 & 46 & 49 \\
\hline $5 / 22$ & 0.56 & 43 & 45 \\
\hline $5 / 23$ & 0.54 & 42 & 44 \\
\hline $5 / 24$ & 0.53 & 42 & 44 \\
\hline $5 / 25$ & 0.56 & 39 & 39 \\
\hline $5 / 26$ & 0.52 & 46 & 43 \\
\hline $5 / 27$ & 0.81 & 31 & 40 \\
\hline $5 / 28$ & 0.75 & 28 & 41 \\
\hline $5 / 29$ & 0.51 & 28 & 34 \\
\hline $5 / 30$ & 0.56 & 28 & 33 \\
\hline $5 / 31$ & 0.64 & 28 & 32 \\
\hline Avg. & 0.81 & 109 & 134 \\
\hline $\operatorname{Max}$. & 4.00 & 451 & 562 \\
\hline $\operatorname{M\perp n}$, & 0.22 & 28 & 30 \\
\hline
\end{tabular}




\section{WSRC-TR-91-624}

INTERNAL DISTRIBUTION LIST:

H.L. DAVIS, 703-41A

K.W. DYER, 742-A

J.L. KEYES, 742-A

C.E. JEWELL, 703-8C

D.W. HAYES, 773-A

J.B. GLADDEN, 773-42A

M.H. PALLER, $773-42 \mathrm{~A}$

E.W. WILDE, 773-42A

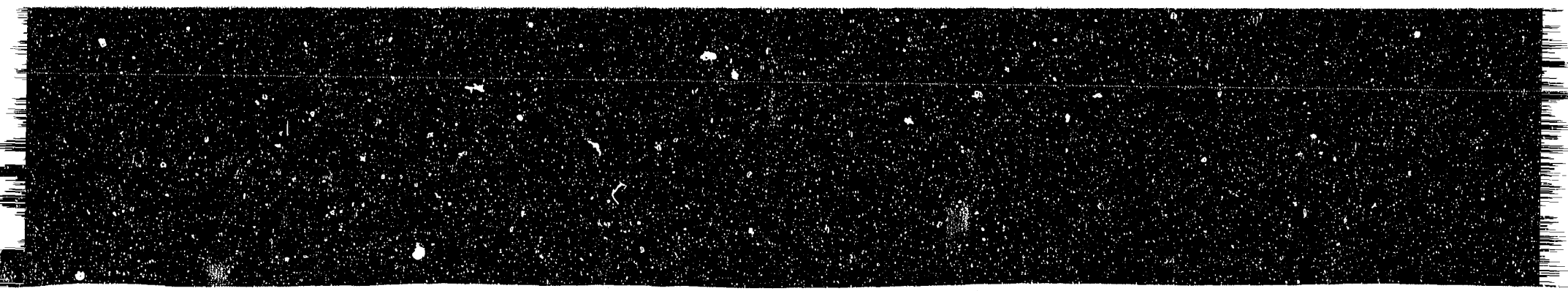

(3)

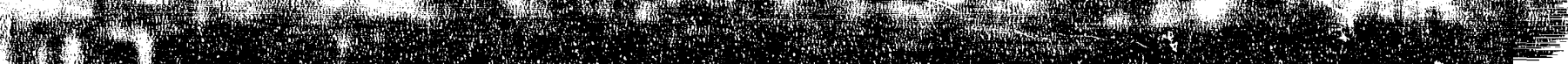



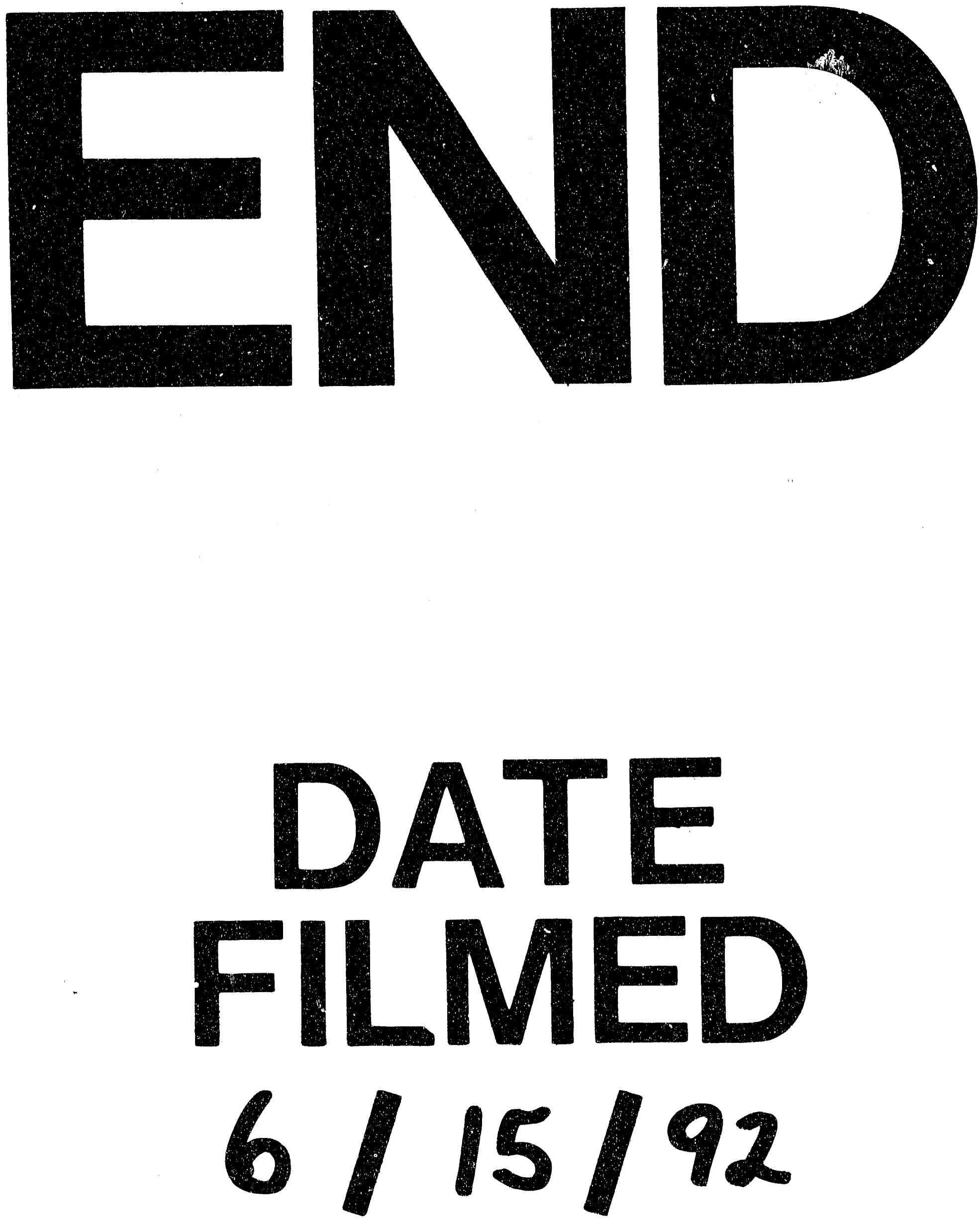

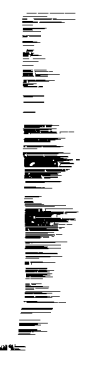




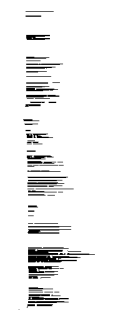

